\title{
Emerging broad-spectrum resistance in Pseudomonas aeruginosa and Acinetobacter baumannii: Mechanisms and epidemiology
}

\author{
Anaïs Potron ${ }^{\mathrm{a}}$, Laurent Poirel ${ }^{\mathrm{b}, *}$, Patrice Nordmann ${ }^{\mathrm{b}, \mathrm{c}}$ \\ a Laboratoire de Bactériologie, Faculté de Médecine-Pharmacie, Centre Hospitalier Régional Universitaire, Université de Franche-Comté, Besançon, France \\ ${ }^{\mathrm{b}}$ Emerging Antibiotic Resistance Medical and Molecular Microbiology Unit, Department of Medicine, Faculty of Science, University of Fribourg, Fribourg, \\ Switzerland \\ ${ }^{\mathrm{c}}$ HFR - Hôpital Cantonal de Fribourg, Fribourg, Switzerland
}

\begin{abstract}
Multidrug resistance is quite common among non-fermenting Gram-negative rods, in particular among clinically relevant species including Pseudomonas aeruginosa and Acinetobacter baumannii. These bacterial species, which are mainly nosocomial pathogens, possess a diversity of resistance mechanisms that may lead to multidrug or even pandrug resistance. Extended-spectrum $\beta$-lactamases (ESBLs) conferring resistance to broad-spectrum cephalosporins, carbapenemases conferring resistance to carbapenems, and 16S rRNA methylases conferring resistance to all clinically relevant aminoglycosides are the most important causes of concern. Concomitant resistance to fluoroquinolones, polymyxins (colistin) and tigecycline may lead to pandrug resistance. The most important mechanisms of resistance in $P$. aeruginosa and $A$. baumannii and their most recent dissemination worldwide are detailed here.
\end{abstract}

\section{Introduction}

The emergence and spread of bacteria resistant to multiple antibiotics and at the origin of severe infections is currently of great concern. This is particularly true for nosocomial pathogens isolated in hospitals, where these superbugs may compromise advanced medicine, including surgery, transplantation, efficient treatment of immunocompromised and haematological patients, etc. Among the increasingly reported and commonly identified multidrug-resistant or even pandrug-resistant bacteria, the lactose-non-fermenting Gram-negative pathogens Acinetobacter baumannii and Pseudomonas aeruginosa occupy an important place. These bacterial species are quick to become multidrug-resistant owing to their additional intrinsic resistance mechanisms. They are responsible for hospital-acquired infections (bloodstream, urinary tract, pulmonary and device-related infections) and are frequently isolated from immunocompromised patients hospitalised in the intensive care unit. Resistance to multiple antibiotic classes, and notably to the $\beta$-lactam cephalosporins and carbapenems, is on the

\footnotetext{
* Corresponding author. Present address: Medical and Molecular Microbiology Unit, Department of Medicine, Faculty of Science, University of Fribourg, rue Albert Gockel 3, CH-1700 Fribourg, Switzerland. Tel.: +41 26300 9582;

fax: +4133670023872 .

E-mail address: laurent.poirel@unifr.ch (L. Poirel).
}

rise worldwide. In this review, the emerging antibiotic resistance mechanisms in A. baumannii and $P$. aeruginosa are highlighted, with a special focus on the most prescribed antimicrobial agents, i.e. $\beta$-lactams, aminoglycosides and fluoroquinolones.

\section{Resistance to $\beta$-lactams}

\subsection{Class A $\beta$-lactamases}

\subsubsection{Extended-spectrum $\beta$-lactamases (ESBLs)}

The class A ESBLs confer resistance to expanded-spectrum cephalosporins and are inhibited in vitro by clavulanic acid and tazobactam [1]. They have been extensively identified in members of the Enterobacteriaceae family but are also reported from non-fermenters.

2.1.1.1. Acinetobacter baumannii. The most common ESBLS described in $A$. baumannii are the PER-, GES- and VEB-type $\beta$ lactamases. The first ESBL identified in A. baumannii was PER-1, being later widely detected in Turkey [2]. PER-1-producing $A$. baumannii are also considered to be widespread in South Korea [3], Hungary [4], Romania [5], Russia [6], Belgium [7] and the USA [8]. They have also been identified in Bulgaria, India, China, Iran and Kuwait [9-13] (Table 1). In A. baumannii, the bla $a_{\mathrm{PER}-1}$ gene is part of a composite transposon named $\operatorname{Tn} 1213$, bracketed by two different insertion sequences (ISPa12 and ISPa13) sharing similar 
Table 1

Ambler class A extended-spectrum $\beta$-lactamases (ESBLs) known in Acinetobacter baumannii and Pseudomonas aeruginosa.

\begin{tabular}{|c|c|c|c|c|}
\hline$\beta$-Lactamase & Host & Genetic support $^{\mathrm{a}}$ & Country of isolation & Reference(s) \\
\hline PER-1 & $\begin{array}{l}\text { Pseudomonas } \\
\text { aeruginosa }\end{array}$ & $\begin{array}{l}\text { C, P } \\
\text { C, P } \\
? \\
\text { C } \\
\text { C } \\
\text { C } \\
? \\
? \\
\text { C } \\
? \\
? \\
\text { P } \\
\text { C } \\
\text { C, P } \\
? \\
\text { C } \\
\text { C } \\
\text { C } \\
\text { P } \\
\text { C } \\
? \\
? \\
? \\
\text { C } \\
?\end{array}$ & $\begin{array}{l}\text { Turkey } \\
\text { South Korea } \\
\text { Hungary } \\
\text { Romania } \\
\text { Russia } \\
\text { Belgium } \\
\text { USA } \\
\text { Bulgaria } \\
\text { India } \\
\text { China } \\
\text { Iran } \\
\text { Kuwait } \\
\text { France } \\
\text { Turkey } \\
\text { Belgium } \\
\text { Italy } \\
\text { Spain } \\
\text { Poland } \\
\text { Hungary } \\
\text { Serbia } \\
\text { Tunisia } \\
\text { Japan } \\
\text { China } \\
\text { Greece } \\
\text { Iran }\end{array}$ & $\begin{array}{l}{[2]} \\
{[3]} \\
{[4]} \\
{[5]} \\
{[6]} \\
{[7]} \\
{[8]} \\
{[9]} \\
{[10]} \\
{[11]} \\
{[12]} \\
{[13]} \\
{[42]} \\
{[2]} \\
{[43]} \\
{[44]} \\
{[45]} \\
{[46]} \\
{[47]} \\
{[47]} \\
{[48]} \\
{[49]} \\
{[50]} \\
{[51]} \\
{[52]}\end{array}$ \\
\hline $\begin{array}{l}\text { PER-2 } \\
\text { PER-3 }\end{array}$ & $\begin{array}{l}\text { Acinetobacter baumannii } \\
\text { Pseudomonas aeruginosa } \\
\text { Acinetobacter baumannii }\end{array}$ & $\begin{array}{l}? \\
? \\
\text { C }\end{array}$ & $\begin{array}{l}\text { Argentina } \\
\text { Bolivia } \\
\text { Egypt }\end{array}$ & $\begin{array}{l}{[15]} \\
{[38]} \\
{[16]}\end{array}$ \\
\hline $\begin{array}{l}\text { PER-7 } \\
\text { PER-8 }\end{array}$ & $\begin{array}{l}\text { Acinetobacter } \\
\text { baumannii } \\
\text { Acinetobacter baumannii }\end{array}$ & $\begin{array}{l}\mathrm{C} \\
\mathrm{P} \\
?\end{array}$ & $\begin{array}{l}\text { France } \\
\text { United Arab Emirates } \\
\text { Nepal }\end{array}$ & $\begin{array}{l}{[17]} \\
{[18]} \\
\text { Accession no. AB985401 }\end{array}$ \\
\hline VEB-2 & $\begin{array}{l}\text { Acinetobacter } \\
\text { baumannii } \\
\text { Pseudomonas } \\
\text { aeruginosa } \\
\text { Pseudomonas aeruginosa }\end{array}$ & $\begin{array}{l}\mathrm{C} \\
\mathrm{C} \\
? \\
? \\
\mathrm{C} \\
\mathrm{C}, \mathrm{P} \\
\mathrm{C}, \mathrm{P} \\
\mathrm{C} \\
? \\
? \\
? \\
? \\
\mathrm{C}\end{array}$ & $\begin{array}{l}\text { France } \\
\text { Belgium } \\
\text { Argentina } \\
\text { Iran } \\
\text { France } \\
\text { Thailand } \\
\text { Kuwait } \\
\text { India } \\
\text { Bulgaria } \\
\text { UK } \\
\text { Denmark } \\
\text { Iran } \\
\text { Thailand }\end{array}$ & $\begin{array}{l}{[19-21]} \\
{[7]} \\
{[15]} \\
{[12]} \\
{[53]} \\
{[54]} \\
{[55]} \\
{[56]} \\
{[57]} \\
{[58]} \\
{[59]} \\
{[12]} \\
{[54]}\end{array}$ \\
\hline $\begin{array}{l}\text { VEB-3 } \\
\text { VEB-7 }\end{array}$ & $\begin{array}{l}\text { Acinetobacter baumannii } \\
\text { Pseudomonas aeruginosa } \\
\text { Acinetobacter baumannii }\end{array}$ & $\begin{array}{l}? \\
? \\
?\end{array}$ & $\begin{array}{l}\text { Taiwan } \\
\text { China } \\
\text { USA }\end{array}$ & $\begin{array}{l}{[23]} \\
{[60]} \\
\text { Accession no. FJ825622 }\end{array}$ \\
\hline $\begin{array}{l}\text { GES-1 } \\
\text { GES-8 (IBC-2) } \\
\text { GES-9 }\end{array}$ & $\begin{array}{l}\text { Pseudomonas } \\
\text { aeruginosa } \\
\text { Pseudomonas aeruginosa } \\
\text { Pseudomonas aeruginosa }\end{array}$ & $\begin{array}{l}\mathrm{C} \\
\mathrm{C} \\
? \\
\mathrm{C} \\
\mathrm{C}\end{array}$ & $\begin{array}{l}\text { France } \\
\text { Brazil } \\
\text { Argentina } \\
\text { Greece } \\
\text { France }\end{array}$ & $\begin{array}{l}{[61]} \\
{[62]} \\
{[63]} \\
{[64]} \\
{[66]}\end{array}$ \\
\hline $\begin{array}{l}\text { GES-12 } \\
\text { GES-13 } \\
\text { GES-22 }\end{array}$ & $\begin{array}{l}\text { Acinetobacter } \\
\text { baumannii } \\
\text { Acinetobacter baumannii } \\
\text { Pseudomonas aeruginosa } \\
\text { Acinetobacter baumannii }\end{array}$ & $\begin{array}{l}\mathrm{P} \\
\mathrm{P} \\
? \\
\mathrm{P} \\
? \\
\mathrm{P} \\
\mathrm{P} \\
\mathrm{C} \\
\mathrm{P}\end{array}$ & $\begin{array}{l}\text { France } \\
\text { Belgium } \\
\text { Sweden } \\
\text { Kuwait } \\
\text { Turkey } \\
\text { Tunisia } \\
\text { Belgium } \\
\text { Greece } \\
\text { Turkey }\end{array}$ & $\begin{array}{l}{[25]} \\
{[26]} \\
{[27]} \\
{[28]} \\
{[29]} \\
{[30]} \\
{[26]} \\
{[65]} \\
{[32]}\end{array}$ \\
\hline SHV-2a & $\begin{array}{l}\text { Pseudomonas } \\
\text { aeruginosa }\end{array}$ & $\begin{array}{l}\mathrm{C} \\
\mathrm{C}\end{array}$ & $\begin{array}{l}\text { France } \\
\text { Tunisia }\end{array}$ & $\begin{array}{l}{[67]} \\
{[68]}\end{array}$ \\
\hline SHV-5 & $\begin{array}{l}\text { Acinetobacter baumannii } \\
\text { Pseudomonas aeruginosa }\end{array}$ & $\begin{array}{l}\mathrm{C} \\
\mathrm{C}\end{array}$ & $\begin{array}{l}\text { France (from USA) } \\
\text { Greece }\end{array}$ & $\begin{array}{l}{[33]} \\
{[71]}\end{array}$ \\
\hline SHV-12 & $\begin{array}{l}\text { Acinetobacter baumannii } \\
\text { Pseudomonas } \\
\text { aeruginosa }\end{array}$ & $\begin{array}{l}\mathrm{P} \\
\mathrm{C} \\
\mathrm{C}\end{array}$ & $\begin{array}{l}\text { The Netherlands } \\
\text { Thailand } \\
\text { Japan }\end{array}$ & $\begin{array}{l}{[35]} \\
{[69]} \\
{[70]}\end{array}$ \\
\hline $\begin{array}{l}\text { TEM-4 } \\
\text { TEM-21 }\end{array}$ & $\begin{array}{l}\text { Pseudomonas aeruginosa } \\
\text { Pseudomonas aeruginosa }\end{array}$ & $\begin{array}{l}\mathrm{C} \\
\mathrm{C}\end{array}$ & $\begin{array}{l}\text { France } \\
\text { France }\end{array}$ & $\begin{array}{l}{[72]} \\
{[73]}\end{array}$ \\
\hline
\end{tabular}


Table 1 (Continued)

\begin{tabular}{|c|c|c|c|c|}
\hline$\beta$-Lactamase & Host & Genetic support ${ }^{\mathrm{a}}$ & Country of isolation & Reference(s) \\
\hline TEM-24 & Pseudomonas aeruginosa & $\mathrm{P}$ & France & [74] \\
\hline TEM-42 & Pseudomonas aeruginosa & $\mathrm{P}$ & France & [75] \\
\hline TEM-92 & Acinetobacter baumannii & ? & Italy & [34] \\
\hline TEM-116 & Acinetobacter baumannii & $\mathrm{P}$ & The Netherlands & [35] \\
\hline CTX-M-1 & Pseudomonas aeruginosa & $?$ & The Netherlands & [76] \\
\hline \multirow[t]{4}{*}{ CTX-M-2 } & Acinetobacter & $\mathrm{P}$ & Japan & [36] \\
\hline & baumannii & $?$ & USA & [37] \\
\hline & Pseudomonas & C & Brazil & {$[77,78]$} \\
\hline & aeruginosa & $?$ & Bolivia & [38] \\
\hline CTX-M-3 & Pseudomonas aeruginosa & $?$ & China & {$[50]$} \\
\hline CTX-M-14 & Pseudomonas aeruginosa & $?$ & China & [50] \\
\hline \multirow[t]{3}{*}{ CTX-M-15 } & Acinetobacter baumannii & $\mathrm{P}$ & India & [39] \\
\hline & & C & Haiti & {$[40]$} \\
\hline & Pseudomonas aeruginosa & $?$ & China & [50] \\
\hline \multirow[t]{2}{*}{ CTX-M-43 } & Acinetobacter baumannii & $?$ & Bolivia & {$[38]$} \\
\hline & Pseudomonas aeruginosa & $?$ & Bolivia & [38] \\
\hline RTG-4 & Acinetobacter baumannii & $\mathrm{P}$ & France & {$[41]$} \\
\hline BEL-1 & Pseudomonas aeruginosa & $\mathrm{C}$ & Belgium & {$[79,80]$} \\
\hline BEL-2 & Pseudomonas aeruginosa & $\mathrm{C}$ & Belgium & [81] \\
\hline BEL-3 & Pseudomonas aeruginosa & $?$ & Spain & [82] \\
\hline PME-1 & Pseudomonas aeruginosa & $\mathrm{P}$ & USA & [83] \\
\hline
\end{tabular}

a C, chromosome; P, plasmid;?, unknown.

inverted repeat sequences [14]. Recently, the bla $a_{\text {PER-1 }}$ gene was identified in a composite transposon made of two copies of ISPa12 in an A. baumannii isolate from Kuwait [13]. PER-2, which is quite distantly related to PER-1 (86\% amino acid identity), has so far been found exclusively in South America [15]. Recently, the bla $a_{\text {PER-3 }}$ gene, initially identified from Aeromonas punctata and Aeromonas caviae, has been identified in a single $A$. baumannii isolate in Egypt [16]. In addition, PER-7 (four amino acid substitutions compared with PER-1) has been identified in a single A. baumannii clinical isolate in France [17] and in the United Arab Emirates (UAE) [18]. The bla $a_{\text {PER-7 }}$ gene was associated with the insertion sequence (IS) element ISCR1 that was also involved it its expression [17]. PER variants identified in A. baumannii are summarised Table 1.

Another important ESBL in A. baumannii is the Vietnamese extended-spectrum $\beta$-lactamase (VEB). VEB- 1 is distantly related to other ESBLs, sharing only $38 \%$ amino acid identity with the closest ESBL, namely PER-1 [19]. VEB-1-producing A. baumannii were first identified in France, where a single clone was originally identified as the source of a hospital outbreak [20]. Genotyping analysis showed that this VEB-1-producing A. baumannii belonged to one of the two major clonal complexes of $A$. baumannii, termed worldwide clone 1 [20]. Further studies showed a nationwide dissemination of VEB-1 in France [21] and its neighbouring country Belgium [7]. In most cases, the bla $a_{\mathrm{VEB}-1}$ gene is identified as a gene cassette in class 1 integrons varying in size and structure [19]. However, in several A. baumannii isolates from Argentina, the bla $a_{\mathrm{VEB}-1}$ gene was associated with an ISCR2 element, which was likely at the origin of the mobilisation of this ESBL gene [22]. VEB-1-producing A. baumannii have also been identified in Iran [12]. The bla $a_{\mathrm{VEB}-3}$ variant was reported in a single $A$. baumannii isolate from Taiwan [23] (Table 1).

Since 2010, GES-type ESBLs are increasingly reported from $A$. baumannii. Actually, GES-1 was firstly reported in 2000, being identified from a single K. pneumoniae isolate [24]. GES-11, differing from GES- 1 by two amino acid substitutions and consequently possessing increased activity towards aztreonam, was first identified in 2009 from an A. baumannii isolate from France [25]. GES-11 was then detected in the same species in Belgium, Sweden, Kuwait, Turkey and Tunisia [26-30] and in the Middle East, which might act as a reservoir for multidrug-resistant bacteria [28]. Another
GES variant, namely GES-12, differs from GES-11 by a single Thr237Ala substitution. It has been identified from several isolates in Belgium [26] and possesses increased hydrolytic activity towards ceftazidime [31]. More recently, GES-22, differing from GES-11 by one amino acid substitution, was reported from two A. baumannii isolates from Turkey [32]. It was reported that GES-22 possessed a hydrolytic profile similar to that of GES-11 and that the bla $a_{\mathrm{GES}-22}$ gene was located on a class 1 integron inserted into a $75-\mathrm{kb}$ plasmid [32].

On the other hand, the TEM- and SHV-type ESBLs, being widespread among Enterobacteriaceae, have been scarcely identified in A. baumannii. The corresponding $b l a_{\mathrm{SHV}}$ and $b l a_{\mathrm{TEM}}$ genes have been identified either on the chromosome $\left(b l a_{\mathrm{SHV}-5}\right)$ or on plasmids (bla $\left.a_{\mathrm{SHV}-12}, b l a_{\mathrm{TEM}-92}, b l a_{\mathrm{TEM}-116}\right)$ [33-35]. Likewise, the genes encoding the CTX-M-type ESBLs, known to be extremely widespread among Enterobacteriaceae, have been rarely identified in A. baumannii. CTX-M-2-producing isolates have been identified in Japan and the USA [36,37], and a CTX-M-43-producing isolate has been found in Bolivia [38]. More recently, CTX-M-15-producing A. baumannii have been identified in India and Haiti $[39,40]$. The bla $_{\text {CTX-M-15 }}$ gene was found to be associated with ISEcp1 in a transposon that integrated into the chromosome of A. baumannii [40]. A novel ESBL, named RTG-4, which is the first carbenicillinase to possess ESBL properties, was identified from an A. baumannii isolate from France in 2009 [41]. This is an atypical ESBL since it significantly hydrolyses cefepime and cefpirome, but hydrolyses ceftazidime only weakly [41].

Although widespread among Enterobacteriaceae, the rare identification of these ESBLs in A. baumannii may be due to limited horizontal gene transfer occurring between these different bacterial genders as a consequence of narrow-spectrum plasmid replication properties.

2.1.1.2. Pseudomonas aeruginosa. The PER-1 $\beta$-lactamase was the first ESBL identified in $P$. aeruginosa [42]. It was identified in a $P$. aeruginosa isolate from a Turkish patient hospitalised in the Paris area of France in 1991 [42]. National surveys from Turkey then showed that PER-1-producing $P$. aeruginosa isolates are widespread in Turkey [2]. PER-1 has been reported in European countries with no geographical border with Turkey, such as Belgium [43], Italy 
[44], Spain [45], Poland [46], Hungary and Serbia [47] and Tunisia [48] as well as in Asian countries [49,50]. In addition, it was identified in Greece and Iran [51,52] (Table 1). Epidemiological surveys have shown that a predominant $P$. aeruginosa sequence type (ST) and single-locus variants, corresponding to international clonal complex CC11, is associated with wide dissemination of PER-1producing $P$. aeruginosa isolates in Turkey, Belgium and Italy as well as in several Eastern European countries [46,47,51]. The PER-2 $\beta$ lactamase, which shares $86 \%$ amino acid identity with PER-1 and therefore represents another lineage of the PER-type enzymes, was identified only from a P. aeruginosa strain isolated in Bolivia [38] (Table 1).

Another ESBL from $P$. aeruginosa is VEB-1, which was identified from $P$. aeruginosa isolates recovered from patients hospitalised in France but transferred from Thailand [53]. Nosocomial spread of VEB-1-producing $P$. aeruginosa isolates was identified in Thailand [54]. Later, other VEB-like producing isolates were reported from Kuwait and India [55,56], but also in Iran, Bulgaria, the UK and Denmark, highlighting the worldwide dissemination of these VEBproducing strains [12,57-59] (Table 1). Isolates producing VEB-2 or VEB-3 were identified in Thailand and China, with these ESBLs differing from VEB-1 by only a single or two amino acid substitutions, respectively $[54,60]$.

Other ESBLs are the GES enzymes, which have been detected in $P$. aeruginosa (Table 2). The bla $a_{\mathrm{GES}-1}$ gene was identified from $P$. aeruginosa isolates from France and South America [61-63]. The structurally related ESBLS IBC-2 (differing from GES-1 by a single amino acid residue and then renamed GES-8) and GES-13 were isolated from two P. aeruginosa isolates in Greece [64,65]. Another variant, named GES-9, possessing a broad-spectrum hydrolysis profile extended to aztreonam, was identified in a single $P$. aeruginosa isolate from France [66].

The SHV-type ESBLs have been identified in very rare isolates of $P$. aeruginosa, being SHV-2a in France and Tunisia $[67,68]$ and SHV-12 in Thailand [69] and Japan [70] (Table 1). A nosocomial outbreak of SHV-5-producing $P$. aeruginosa was also described in Greece [71]. TEM-type ESBLs have also been rarely reported from $P$. aeruginosa, being TEM-4 [72], TEM-21 [73], TEM-24 [74] and TEM42 [75]. CTX-M-type ESBLs, with in some cases evidence of their horizontal transfer from Enterobacteriaceae to $P$. aeruginosa, are very rarely identified in $P$. aeruginosa. A single CTX-M-1-producing $P$. aeruginosa isolate has been reported from The Netherlands in 2006 [76], and CTX-M-2- or CTX-M-43-positive P. aeruginosa have been identified in South America [38,77,78]. Recently, CTX-M-3, CTX-M-14 and CTX-M-15 were identified from several P. aeruginosa isolates from China [50] (Table 1).

In 2005, another ESBL that had weak amino acid identity with other class A ESBLs but had similar biochemical properties was reported; the gene encoding BEL-1 was located in a class 1 integron inserted into the chromosome of a $P$. aeruginosa isolate recovered in a single hospital in Belgium [79]. Later, another study reported the dissemination of BEL-1-producing $P$. aeruginosa isolates in several hospitals located in different geographical areas in Belgium [80]. BEL-2 and BEL-3, each differing from BEL-1 by a single amino acid substitution, were identified in 2010 in a strain recovered in Belgium and Spain, respectively $[81,82]$ (Table 1 ). Compared with BEL-1, BEL-2 possesses enhanced hydrolytic properties against expanded-spectrum cephalosporins [81].

The ESBL PME-1 is the latest identified ESBL from a clinical $P$. aeruginosa isolate and was recovered in Pennsylvania, USA, in 2008 [83]. This enzyme shares $43 \%$ amino acid identity with the closest ESBL CTX-M-9. PME-1 confers a high level of resistance to penicillins, ceftazidime and aztreonam and to a lesser extent cefotaxime, but spares cefepime and the carbapenems. The bla $a_{\mathrm{PME}-1}$ gene was found to be located on an ca. 9-kb plasmid, flanked on both extremities by two copies of ISCR24 [83].

\subsubsection{Carbapenemases}

2.1.2.1. Acinetobacter baumannii. Although almost all class A ESBLs do not possess any significant carbapenemase activity, specific GES variants have been shown to possess the ability to compromise the efficacy of carbapenems (Table 2). These are GES enzymes possessing specific residues enlarging their hydrolysis spectrum, and some of them such as GES-5 have been identified in Enterobacteriaceae [84]. The GES-14 variant is one of these GES-type carbapenemases and has been identified in A. baumannii in France in 2011 [85], the $b l a_{\mathrm{GES}-14}$ gene being part of a class 1 integron located on a selftransferable plasmid [85].

Another class A carbapenemase that is commonly identified among Enterobacteriaceae is KPC, with KPC-type enzymes possessing intrinsic high carbapenemase and ESBL activity [86]. These enzymes all confer resistance to all $\beta$-lactams, and the corresponding genes are located on mobile genetic elements, enhancing their spread [87]. Despite wide dissemination among enterobacterial species, only a few KPC-type $\beta$-lactamases have been identified in A. baumannii, being from a series of isolates recovered in Puerto Rico [88]. In that study, ten A. baumannii isolates producing KPC-type enzymes were detected, corresponding to KPC-3 (7 isolates) and KPC-2, KPC-4 and KPC-10 in single isolates, respectively [88].

2.1.2.2. Pseudomonas aeruginosa. As highlighted earlier, several GES-type ESBLs exhibit some carbapenemase properties. Actually, the first GES-type carbapenemase was identified from a $P$. aeruginosa isolate, being GES-2, differing from GES-1 by a single amino acid substitution [89]. This isolate was recovered from a patient hospitalised in South Africa and was actually part of an outbreak that occurred in the same hospital [90]. The GES-5 variant possessing significant carbapenemase activity has also been reported from $P$. aeruginosa isolates in China [91], South Africa [92], Brazil [93] and Turkey [94]. These bla $_{\mathrm{GES}}$-type genes are part of class 1 integron structures [92]. Recently, a novel GES variant, GES-18, was identified from a P. aeruginosa isolate from Belgium. GES-18 differed from GES-5 by one amino acid substitution and also hydrolysed carbapenems [95].

Although rarely identified, KPC-producing $P$. aeruginosa isolates have been reported, first in Colombia in 2006 [96] and then in Puerto Rico [97,98], Trinidad and Tobago [99], the USA [100] and China [101]. They are increasingly identified in the Americas and the Caribbean region [102-104]. No clear evidence of horizontal transfer of the $b l a_{\mathrm{KPC}}$ gene from Enterobacteriaceae to non-fermenters has been observed.

\subsection{Class B $\beta$-lactamases}

These $\beta$-lactamases, also named metallo- $\beta$-lactamases (MBLs), hydrolyse carbapenems and other $\beta$-lactams (except monobactams) very efficiently and they are not inhibited by the clinically available $\beta$-lactamase inhibitors such as clavulanic acid or tazobactam. However, their activity is inhibited by metal ion chelators $[105,106]$.

\subsubsection{Acinetobacter baumannii}

Carbapenem resistance in this species is most often (if not always) linked to the production of carbapenemases. MBL enzymes are not the most commonly identified carbapenemases in A. baumannii; when identified, they are either IMP-like, VIM-like, SIM-1 or NDM-like enzymes [107]. Nine IMP variants have been identified in A. baumannii, namely IMP-1 in Italy [108], Japan [109], South Korea [110], India [111], Taiwan [112] and Kuwait [113], IMP-2 in Japan and Italy [109,114], IMP-4 in Hong-Kong [115], Australia and Singapore [116,117], IMP-5 in Portugal [118], IMP-6 in Brazil [119], IMP-8 in China [120], IMP-11 in Japan (accession no. AB074436), 
Table 2

Ambler class A carbapenemases known in Acinetobacter baumannii and Pseudomonas aeruginosa.

\begin{tabular}{|c|c|c|c|c|}
\hline$\beta$-Lactamase & Host & Genetic support ${ }^{\mathrm{a}}$ & Country of isolation & Reference(s) \\
\hline GES-2 & Pseudomonas aeruginosa & $\mathrm{P}$ & South Africa & {$[89,90]$} \\
\hline GES-5 & $\begin{array}{l}\text { Pseudomonas } \\
\text { aeruginosa }\end{array}$ & $\begin{array}{l}? \\
? \\
\text { C } \\
?\end{array}$ & $\begin{array}{l}\text { China } \\
\text { South Africa } \\
\text { Brazil } \\
\text { Turkey }\end{array}$ & $\begin{array}{l}{[91]} \\
{[92]} \\
{[93]} \\
{[94]}\end{array}$ \\
\hline $\begin{array}{l}\text { GES-14 } \\
\text { GES-18 }\end{array}$ & $\begin{array}{l}\text { Acinetobacter baumannii } \\
\text { Pseudomonas aeruginosa }\end{array}$ & $\begin{array}{l}\mathrm{P} \\
\mathrm{C}\end{array}$ & $\begin{array}{l}\text { France } \\
\text { Belgium }\end{array}$ & $\begin{array}{l}{[85]} \\
{[95]}\end{array}$ \\
\hline KPC-2 & $\begin{array}{l}\text { Acinetobacter baumannii } \\
\text { Pseudomonas } \\
\text { aeruginosa }\end{array}$ & $\begin{array}{l}? \\
\mathrm{C}, \mathrm{P} \\
? \\
? \\
\mathrm{P} \\
\mathrm{C} \\
? \\
?\end{array}$ & $\begin{array}{l}\text { Puerto Rico } \\
\text { Columbia } \\
\text { Puerto Rico } \\
\text { Trinidad and Tobago } \\
\text { USA } \\
\text { China } \\
\text { Argentina } \\
\text { Brazil }\end{array}$ & $\begin{array}{l}{[88]} \\
{[96,102]} \\
{[97]} \\
{[99]} \\
{[100]} \\
{[101]} \\
{[103]} \\
{[104]}\end{array}$ \\
\hline KPC-3 & Acinetobacter baumannii & $?$ & Puerto Rico & {$[88]$} \\
\hline KPC-4 & Acinetobacter baumannii & $?$ & Puerto Rico & [88] \\
\hline KPC-5 & Pseudomonas aeruginosa & $?$ & Puerto Rico & [98] \\
\hline KPC-10 & Acinetobacter baumannii & $?$ & Puerto Rico & [88] \\
\hline
\end{tabular}

a C, chromosome; P, plasmid;?, unknown.

IMP-14 in Thailand [121] and IMP-19 in Japan [122] (Table 3). Noteworthy, VIM-type enzymes that have been widely identified in Enterobacteriaceae have rarely been identified in A. baumannii. There are few reports of VIM-1-producers in Greece [123], VIM-2 in South Korea [110] and Kuwait [113], VIM-4 in Italy [124], VIM6 in India (accession no. EF645347) and VIM-11 in Taiwan [23] (Table 3).

The SIM-1 carbapenemase has been reported only in the A. baumannii species so far, and only in South Korea, where this resistance trait appears to be widespread [125]. Analysis of the genetic support of the MBL-encoding genes identified in A. baumannii shows similar structures, with the $b l a_{\mathrm{IMP}}, b l a_{\mathrm{VIM}}$ and $b l a_{\mathrm{SIM}}$ genes being all embedded in class 1 integron structures [107].

NDM- 1 is one of the most recently identified MBLs [126]. Whilst most studies indicate wide dissemination of the $b l a_{\mathrm{NDM}-1}$-like genes in Enterobacteriaceae, many studies reported on the acquisition of bla $a_{\mathrm{NDM}-1}$-like genes in A. baumannii. Indeed, NDM-1 was first reported in India from Enterobacteriaceae and then in A. baumannii $[126,127]$. Other reports are from different European countries and from China, Japan, Kenya, Brazil, Algeria and Syria [128-137]. An outbreak of NDM-1-producing A. baumannii, belonging to ST85, was recently reported in France [138], underscoring the growing concern related to the spread of these isolates in Europe. Identification of several ST85 isolates possessing the $b l a_{\mathrm{NDM}-1}$ gene and originating from North Africa, with no obvious link to the Indian subcontinent, strongly suggests that the source of NDM-producing A. baumannii strains could be North Africa [139]. Another variant, NDM-2, was identified in A. baumannii strains recovered in Egypt [140], Israel [141] and the UAE [142]. Interestingly, it was evidenced that these NDM-2-producing isolates were clonally related, suggesting that the Middle East as well as the Balkan region and the Indian and China regions might act as reservoirs of NDM-2producing Acinetobacter [143]. In these isolates, the bla NDM gene was surrounded by two copies of ISAba125, thus forming a 10099 bp composite transposon named Tn125 [144]. As opposed to what is observed in Enterobacteriaceae, the ISAba125 element located upstream of bla $a_{\mathrm{NDM}}$ and that plays a role in its expression, is not truncated [144]. Our extensive studies showed that $A$. baumannii was likely the first target of bla $a_{\mathrm{NDM}-1}$ gene acquisition before its transfer to Enterobacteriaceae and P. aeruginosa [144]. This represents a new paradigm in antibiotic resistance since it highlights that Acinetobacter spp. may be a source of an important resistance trait for Enterobacteriaceae.

\subsubsection{Pseudomonas aeruginosa}

Carbapenem resistance in $P$. aeruginosa is mostly related to porin (OprD) deficiency and more rarely to carbapenemases. Carbapenemases in $P$. aeruginosa are mainly MBLs of the IMP, VIM, SPM and GIM types. IMP-1 was first reported in Enterobacteriaceae and $P$. aeruginosa in Japan and is now globally distributed, suggesting

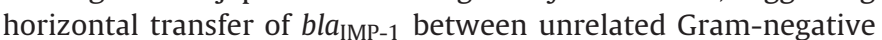
species [145]. IMP-like enzymes may be divided into several subgroups and the percentage amino acid identity within these groups actually ranges from $90 \%$ to $99 \%$ [106]. These variants possess very similar hydrolytic activities. Among the 51 known IMP variants, 32 have been reported from $P$. aeruginosa and have been identified throughout the world (Table 3).

Although VIM enzymes share $<40 \%$ amino acid identity with the IMP-type enzymes, they share the same hydrolytic spectrum [186]. VIM-1 was the first MBL identified in P. aeruginosa [187] and has been reported in several European countries (Table 3). However, VIM-2 is now the most widespread MBL in P. aeruginosa as a source of multiple outbreaks [106]. Twenty-three of the forty-six VIM variants have been identified in P. aeruginosa (Table 3).

$\beta$-Lactamase SPM is quite different from VIM and IMP and, accordingly, represents a new subfamily of MBLs. SPM- 1 was first isolated in Brazil in 1997 from a P. aeruginosa clinical isolate [207], which was highly resistant to all anti-Gram-negative antibiotics except colistin. Dissemination of multidrug-resistant $P$. aeruginosa producing SPM-1 was demonstrated in distinct regions of this country, however they have not disseminated in other countries [208], with the only exception of a single isolate identified in Switzerland from a patient who had previously been hospitalised in Brazil [209]. The bla $a_{\mathrm{SPM}-1}$ gene is either chromosomal or plasmidencoded. In addition, it is associated with the IS element ISCR4 at the origin of its acquisition and expression and is likely transposed through a rolling-circle replication mechanism [210].

In 2002, a new type of acquired MBL, named GIM-1, was identified in clonally related $P$. aeruginosa isolates from Germany $[211,212]$. This enzyme also produced by enterobacterial species has only been identified in Germany.

NDM-1-producing $P$. aeruginosa isolates were first reported in 2011, with two strains recovered from Serbia [213]. In 2012, a single NDM-1-producing $P$. aeruginosa belonging to ST235 was isolated in France from a patient previously hospitalised in Serbia [214,215]. More recently, NDM-1-positive $P$. aeruginosa isolates were recovered in India (four isolates), Italy (a single isolate belonging to 
Table 3

Ambler class B metallo- $\beta$-lactamases known in Acinetobacter baumannii and Pseudomonas aeruginosa.

\begin{tabular}{|c|c|c|c|c|}
\hline$\beta$-Lactamase & Host & Genetic environment or support ${ }^{\mathrm{a}}$ & Country of isolation & Reference(s) \\
\hline IMP-1 & $\begin{array}{l}\text { Pseudomonas } \\
\text { aeruginosa }\end{array}$ & $\begin{array}{l}? \\
\text { I } \\
? \\
? \\
? \\
? \\
\text { I } \\
\text { I } \\
? \\
? \\
\text { I } \\
? \\
\text { I }\end{array}$ & $\begin{array}{l}\text { Italy } \\
\text { Japan } \\
\text { South Korea } \\
\text { India } \\
\text { Taiwan } \\
\text { Kuwait } \\
\text { Japan } \\
\text { South Korea } \\
\text { Brazil } \\
\text { China } \\
\text { Turkey } \\
\text { Singapore } \\
\text { Thailand }\end{array}$ & $\begin{array}{l}{[108]} \\
{[109]} \\
{[110]} \\
{[111]} \\
{[112]} \\
{[113]} \\
{[146]} \\
{[110]} \\
{[147]} \\
{[148]} \\
{[149]} \\
{[150]} \\
{[151]}\end{array}$ \\
\hline IMP-2 & $\begin{array}{l}\text { Acinetobacter } \\
\text { baumannii } \\
\text { Pseudomonas aeruginosa }\end{array}$ & $\begin{array}{l}\text { I } \\
\text { I } \\
\text { I }\end{array}$ & $\begin{array}{l}\text { Japan } \\
\text { Italy } \\
\text { Japan }\end{array}$ & $\begin{array}{l}{[109]} \\
{[114]} \\
{[109]}\end{array}$ \\
\hline IMP-4 & $\begin{array}{l}\text { Acinetobacter } \\
\text { baumannii } \\
\text { Pseudomonas } \\
\text { aeruginosa }\end{array}$ & $\begin{array}{l}\mathrm{I} \\
? \\
\mathrm{I} \\
? \\
\mathrm{I}\end{array}$ & $\begin{array}{l}\text { Hong Kong } \\
\text { Australia } \\
\text { Singapore } \\
\text { Malaysia } \\
\text { Australia }\end{array}$ & $\begin{array}{l}{[115]} \\
{[116]} \\
{[117]} \\
{[152]} \\
{[153]}\end{array}$ \\
\hline IMP-5 & $\begin{array}{l}\text { Acinetobacter baumannii } \\
\text { Pseudomonas aeruginosa }\end{array}$ & $\begin{array}{l}\text { I } \\
\text { I }\end{array}$ & $\begin{array}{l}\text { Portugal } \\
\text { Portugal }\end{array}$ & $\begin{array}{l}{[118]} \\
{[154]}\end{array}$ \\
\hline IMP-6 & $\begin{array}{l}\text { Acinetobacter baumannii } \\
\text { Pseudomonas } \\
\text { aeruginosa }\end{array}$ & $\begin{array}{l}? \\
\text { I } \\
?\end{array}$ & $\begin{array}{l}\text { Brazil } \\
\text { South Korea } \\
\text { China }\end{array}$ & $\begin{array}{l}{[119]} \\
{[155]} \\
{[156]}\end{array}$ \\
\hline IMP-7 & $\begin{array}{l}\text { Pseudomonas } \\
\text { aeruginosa }\end{array}$ & $\begin{array}{l}\text { I } \\
? \\
? \\
\text { I } \\
? \\
\text { I } \\
?\end{array}$ & $\begin{array}{l}\text { Canada } \\
\text { Malaysia } \\
\text { Slovakia } \\
\text { Japan } \\
\text { Singapore } \\
\text { Czech Republic } \\
\text { Denmark }\end{array}$ & $\begin{array}{l}{[157]} \\
{[158]} \\
{[159]} \\
{[160]} \\
{[150]} \\
{[161]} \\
{[162]}\end{array}$ \\
\hline $\begin{array}{l}\text { IMP-8 } \\
\text { IMP-9 } \\
\text { IMP-10 }\end{array}$ & $\begin{array}{l}\text { Acinetobacter baumannii } \\
\text { Pseudomonas aeruginosa } \\
\text { Pseudomonas aeruginosa }\end{array}$ & $\begin{array}{l}\text { I } \\
\text { I } \\
\text { I }\end{array}$ & $\begin{array}{l}\text { China } \\
\text { China } \\
\text { Japan }\end{array}$ & $\begin{array}{l}{[120]} \\
{[163]} \\
{[164]}\end{array}$ \\
\hline IMP-11 & $\begin{array}{l}\text { Acinetobacter baumannii } \\
\text { Pseudomonas aeruginosa }\end{array}$ & $\begin{array}{l}? \\
?\end{array}$ & $\begin{array}{l}\text { Japan } \\
\text { Japan }\end{array}$ & $\begin{array}{l}\text { Accession no. AB074436 } \\
\text { Accession no. AB074437 }\end{array}$ \\
\hline IMP-13 & $\begin{array}{l}\text { Pseudomonas } \\
\text { aeruginosa }\end{array}$ & $\begin{array}{l}\text { I } \\
\text { I } \\
\text { I } \\
\text { I }\end{array}$ & $\begin{array}{l}\text { Austria } \\
\text { Italy } \\
\text { France } \\
\text { Belgium }\end{array}$ & $\begin{array}{l}{[165]} \\
{[166]} \\
{[167]} \\
{[168]}\end{array}$ \\
\hline IMP-14 & $\begin{array}{l}\text { Acinetobacter baumannii } \\
\text { Pseudomonas aeruginosa }\end{array}$ & $\begin{array}{l}\mathrm{I} \\
\mathrm{I}\end{array}$ & $\begin{array}{l}\text { Thailand } \\
\text { Thailand }\end{array}$ & $\begin{array}{l}{[121]} \\
{[169]}\end{array}$ \\
\hline IMP-16 & $\begin{array}{l}\text { Pseudomonas } \\
\text { aeruginosa } \\
\text { Pseudomonas aeruginosa }\end{array}$ & $\begin{array}{l}\text { I } \\
\text { I } \\
? \\
\text { I }\end{array}$ & $\begin{array}{l}\text { Mexico } \\
\text { Spain } \\
\text { Germany } \\
\text { Brazil }\end{array}$ & $\begin{array}{l}{[170]} \\
{[171]} \\
{[172]} \\
{[173]}\end{array}$ \\
\hline IMP-18 & $\begin{array}{l}\text { Pseudomonas } \\
\text { aeruginosa }\end{array}$ & $\begin{array}{l}? \\
\text { I } \\
\text { I }\end{array}$ & $\begin{array}{l}\text { USA } \\
\text { Mexico } \\
\text { Puerto Rico }\end{array}$ & $\begin{array}{l}{[174]} \\
{[175]} \\
{[97]}\end{array}$ \\
\hline $\begin{array}{l}\text { IMP-20 } \\
\text { IMP-21 }\end{array}$ & $\begin{array}{l}\text { Acinetobacter baumannii } \\
\text { Pseudomonas } \\
\text { aeruginosa } \\
\text { Pseudomonas aeruginosa } \\
\text { Pseudomonas aeruginosa }\end{array}$ & $\begin{array}{l}? \\
? \\
\text { I } \\
\text { I }\end{array}$ & $\begin{array}{l}\text { Japan } \\
\text { Japan } \\
\text { Italy } \\
\text { Japan } \\
\text { Japan }\end{array}$ & $\begin{array}{l}{[122]} \\
\text { Accession no. AB184876 } \\
{[176]} \\
\text { Accession no. AB196988 } \\
\text { Accession no. AB204557 }\end{array}$ \\
\hline $\begin{array}{l}\text { IMP-22 } \\
\text { IMP-25 }\end{array}$ & $\begin{array}{l}\text { Pseudomonas } \\
\text { aeruginosa } \\
\text { Pseudomonas aeruginosa }\end{array}$ & $\begin{array}{l}\text { I } \\
\text { I } \\
\text { I }\end{array}$ & $\begin{array}{l}\text { Austria } \\
\text { Italy } \\
\text { China }\end{array}$ & $\begin{array}{l}{[165]} \\
{[177]} \\
\text { Accession no. EU352796 }\end{array}$ \\
\hline IMP-26 & $\begin{array}{l}\text { Pseudomonas } \\
\text { aeruginosa }\end{array}$ & $\begin{array}{l}\text { I } \\
?\end{array}$ & $\begin{array}{l}\text { Malaysia } \\
\text { Singapore }\end{array}$ & $\begin{array}{l}{[178]} \\
{[179]}\end{array}$ \\
\hline IMP-29 & Pseudomonas aeruginosa & I & France & {$[180]$} \\
\hline $\begin{array}{l}\text { IMP-30 } \\
\text { IMP-31 }\end{array}$ & $\begin{array}{l}\text { Pseudomonas aeruginosa } \\
\text { Pseudomonas aeruginosa }\end{array}$ & $\begin{array}{l}? \\
?\end{array}$ & $\begin{array}{l}\text { Russia } \\
\text { Germany }\end{array}$ & $\begin{array}{l}{[181]} \\
\text { Accession no. KF148593 }\end{array}$ \\
\hline IMP-33 & Pseudomonas aeruginosa & I & Italy & {$[182]$} \\
\hline IMP-35 & Pseudomonas aeruginosa & $\mathrm{I}$ & Germany & [183] \\
\hline IMP-37 & Pseudomonas aeruginosa & $?$ & France & Accession no. JX131372 \\
\hline IMP-40 & Pseudomonas aeruginosa & $?$ & Japan & Accession no. AB753457 \\
\hline
\end{tabular}


Table 3 (Continued)

\begin{tabular}{|c|c|c|c|c|}
\hline$\beta$-Lactamase & Host & Genetic environment or support ${ }^{\mathrm{a}}$ & Country of isolation & Reference(s) \\
\hline IMP-41 & Pseudomonas aeruginosa & $?$ & Japan & Accession no. AB753458 \\
\hline IMP-43 & Pseudomonas aeruginosa & I & Japan & {$[184]$} \\
\hline IMP-44 & Pseudomonas aeruginosa & $\mathrm{I}$ & Japan & [184] \\
\hline IMP-45 & Pseudomonas aeruginosa & I & China & [185] \\
\hline IMP-48 & Pseudomonas aeruginosa & $?$ & USA & Accession no. KM087857 \\
\hline VIM-1 & $\begin{array}{l}\text { Acinetobacter baumannii } \\
\text { Pseudomonas } \\
\text { aeruginosa }\end{array}$ & $\begin{array}{l}\text { I } \\
\text { I } \\
\text { I } \\
\text { I } \\
? \\
\text { I }\end{array}$ & $\begin{array}{l}\text { Greece } \\
\text { Italy } \\
\text { France } \\
\text { Greece } \\
\text { Germany } \\
\text { Italy }\end{array}$ & $\begin{array}{l}{[123]} \\
{[187]} \\
{[188]} \\
{[189]} \\
{[172]} \\
{[176]}\end{array}$ \\
\hline VIM-2 & $\begin{array}{l}\text { Acinetobacter } \\
\text { baumannii } \\
\text { Pseudomonas } \\
\text { aeruginosa }\end{array}$ & $\begin{array}{l}\text { I } \\
? \\
\text { I } \\
? \\
\text { I } \\
\text { I } \\
? \\
? \\
\text { I } \\
\text { I } \\
\text { I } \\
\text { I } \\
\text { I } \\
\text { I } \\
\text { I } \\
\text { I } \\
? \\
\text { I } \\
\text { I } \\
\text { I } \\
\text { I } \\
? \\
\text { I } \\
? \\
? \\
?\end{array}$ & $\begin{array}{l}\text { South Korea } \\
\text { Kuwait } \\
\text { Tunisia } \\
\text { Thailand } \\
\text { Austria } \\
\text { Mexico } \\
\text { India } \\
\text { Kenya } \\
\text { Hungary } \\
\text { Malaysia } \\
\text { South Korea } \\
\text { Japan } \\
\text { France } \\
\text { Greece } \\
\text { Italy } \\
\text { Portugal } \\
\text { Spain } \\
\text { Croatia } \\
\text { Poland } \\
\text { Chile } \\
\text { Venezuela } \\
\text { Argentina } \\
\text { USA } \\
\text { Belgium } \\
\text { Germany } \\
\text { Turkey }\end{array}$ & $\begin{array}{l}{[110]} \\
{[113]} \\
{[190]} \\
{[169]} \\
{[165]} \\
{[170]} \\
{[191]} \\
{[192]} \\
{[193]} \\
{[194]} \\
{[106]} \\
{[106]} \\
{[106]} \\
{[106]} \\
{[106]} \\
{[106]} \\
{[106]} \\
{[106]} \\
{[106]} \\
{[106]} \\
{[106]} \\
{[106]} \\
{[106]} \\
{[172]} \\
{[172]} \\
{[172]}\end{array}$ \\
\hline VIM-3 & Pseudomonas aeruginosa & $?$ & Taiwan & [195] \\
\hline VIM-4 & $\begin{array}{l}\text { Acinetobacter baumannii } \\
\text { Pseudomonas } \\
\text { aeruginosa }\end{array}$ & $\begin{array}{l}? \\
\text { I } \\
? \\
\text { I } \\
\text { I } \\
?\end{array}$ & $\begin{array}{l}\text { Italy } \\
\text { Greece } \\
\text { Sweden } \\
\text { Poland } \\
\text { Hungary } \\
\text { France }\end{array}$ & $\begin{array}{l}{[124]} \\
{[106]} \\
{[106]} \\
{[106]} \\
{[193,196]} \\
{[172]}\end{array}$ \\
\hline VIM-5 & $\begin{array}{l}\text { Pseudomonas } \\
\text { aeruginosa }\end{array}$ & $\begin{array}{l}? \\
\text { I }\end{array}$ & $\begin{array}{l}\text { India } \\
\text { Turkey }\end{array}$ & $\begin{array}{l}{[191]} \\
{[106]}\end{array}$ \\
\hline VIM-6 & $\begin{array}{l}\text { Acinetobacter baumannii } \\
\text { Pseudomonas } \\
\text { aeruginosa }\end{array}$ & $\begin{array}{l}? \\
\text { I } \\
\text { I } \\
\text { I } \\
\text { I }\end{array}$ & $\begin{array}{l}\text { India } \\
\text { India } \\
\text { Indonesia } \\
\text { South Korea } \\
\text { Philippines }\end{array}$ & $\begin{array}{l}\text { Accession no. EF645347 } \\
{[191]} \\
{[197]} \\
{[197]} \\
{[197]}\end{array}$ \\
\hline VIM-7 & Pseudomonas aeruginosa & I & USA & [198] \\
\hline VIM-8 & Pseudomonas aeruginosa & $?$ & Columbia & [199] \\
\hline $\begin{array}{l}\text { VIM-9 } \\
\text { VIM-10 }\end{array}$ & $\begin{array}{l}\text { Pseudomonas aeruginosa } \\
\text { Pseudomonas aeruginosa }\end{array}$ & $\begin{array}{l}? \\
?\end{array}$ & $\begin{array}{l}\text { UK } \\
\text { UK }\end{array}$ & $\begin{array}{l}\text { Accession no. AY524988 } \\
{[58]}\end{array}$ \\
\hline VIM-11 & $\begin{array}{l}\text { Acinetobacter baumannii } \\
\text { Pseudomonas } \\
\text { aeruginosa }\end{array}$ & $\begin{array}{l}\text { I } \\
\text { I } \\
? \\
? \\
\text { I }\end{array}$ & $\begin{array}{l}\text { Taiwan } \\
\text { India } \\
\text { Argentina } \\
\text { Italy } \\
\text { Malaysia }\end{array}$ & $\begin{array}{l}{[23]} \\
{[191]} \\
{[200]} \\
\text { Accession no. AY635904 } \\
{[194]}\end{array}$ \\
\hline VIM-13 & Pseudomonas aeruginosa & I & Spain & [201] \\
\hline VIM-14 & $\begin{array}{l}\text { Pseudomonas } \\
\text { aeruginosa }\end{array}$ & $\begin{array}{l}? \\
\text { I }\end{array}$ & $\begin{array}{l}\text { Spain } \\
\text { Italy }\end{array}$ & $\begin{array}{l}\text { Accession no. EF055455 } \\
{[202]}\end{array}$ \\
\hline VIM-15 & Pseudomonas aeruginosa & I & Bulgaria & [203] \\
\hline VIM-16 & Pseudomonas aeruginosa & I & Germany & [203] \\
\hline VIM-17 & Pseudomonas aeruginosa & I & Greece & [204] \\
\hline VIM-18 & Pseudomonas aeruginosa & I & India & [191] \\
\hline VIM-20 & Pseudomonas aeruginosa & $?$ & Spain & [205] \\
\hline VIM-28 & Pseudomonas aeruginosa & I & Egypt & [206] \\
\hline VIM-30 & Pseudomonas aeruginosa & I & France & Accession no. JN129451 \\
\hline VIM-36 & Pseudomonas aeruginosa & $?$ & Belgium & {$[172]$} \\
\hline
\end{tabular}


Table 3 (Continued)

\begin{tabular}{|c|c|c|c|c|}
\hline$\beta$-Lactamase & Host & Genetic environment or support ${ }^{\mathrm{a}}$ & Country of isolation & Reference(s) \\
\hline VIM-37 & Pseudomonas aeruginosa & $?$ & Poland & {$[172]$} \\
\hline VIM-38 & Pseudomonas aeruginosa & I & Turkey & {$[94]$} \\
\hline SIM-1 & Acinetobacter baumannii & I & South Korea & [125] \\
\hline \multirow[t]{19}{*}{ NDM-1 } & \multirow{13}{*}{$\begin{array}{l}\text { Acinetobacter } \\
\text { baumannii }\end{array}$} & C & Czech Republic & [128] \\
\hline & & $\mathrm{C}$ & Germany & {$[129,131]$} \\
\hline & & C & Belgium & [130] \\
\hline & & $\mathrm{C}$ & Slovenia & [131] \\
\hline & & $\mathrm{C}$ & France & [131] \\
\hline & & C & Switzerland & [131] \\
\hline & & $?$ & India & [127] \\
\hline & & $\mathrm{P}$ & China & [132] \\
\hline & & $?$ & Japan & [133] \\
\hline & & $?$ & Kenya & {$[134]$} \\
\hline & & $\mathrm{P}$ & Brazil & [135] \\
\hline & & $?$ & Algeria & [136] \\
\hline & & $?$ & Syria & [137] \\
\hline & \multirow{6}{*}{$\begin{array}{l}\text { Pseudomonas } \\
\text { aeruginosa }\end{array}$} & C & Serbia & [213] \\
\hline & & $\mathrm{C}$ & France & {$[214,215]$} \\
\hline & & $\mathrm{P}$ & India & {$[216]$} \\
\hline & & C & Italy & [217] \\
\hline & & $?$ & Egypt & [218] \\
\hline & & $?$ & Slovakia & [219] \\
\hline \multirow[t]{3}{*}{ NDM-2 } & \multirow{3}{*}{$\begin{array}{l}\text { Acinetobacter } \\
\text { baumannii }\end{array}$} & C & Egypt & {$[140]$} \\
\hline & & $\mathrm{C}$ & Israel & [141] \\
\hline & & $?$ & United Arab Emirates & [142] \\
\hline \multirow[t]{2}{*}{ SPM-1 } & \multirow{2}{*}{$\begin{array}{l}\text { Pseudomonas } \\
\text { aeruginosa }\end{array}$} & ISCR4 & Brazil & [207] \\
\hline & & ISCR4 & Switzerland & [209] \\
\hline GIM-1 & Pseudomonas aeruginosa & I & Germany & {$[211,212]$} \\
\hline FIM-1 & Pseudomonas aeruginosa & C & Italy & {$[221]$} \\
\hline
\end{tabular}

${ }^{\text {a }}$ I, integron present; C, chromosome; P, plasmid;?, unknown.

ST235), Egypt and Slovakia [216-219]. Interestingly, the emergence of multidrug-resistant VIM-2-producing $P$. aeruginosa in Russia is also linked to a ST235-like dominant clone [220]. Association of ST235-like strains with MBL genes has been reported in several European countries [220], such as in Italy with VIM-1-producers, in Greece, Sweden, Hungary and Belgium with VIM-4-producers, in Spain with VIM-13-producers, and in France with IMP-29producers [220]. This clone might therefore possess some specific traits enhancing its clonal dissemination.

Recently, a novel MBL named FIM-1, exhibiting its highest similarity (40\% amino acid identity) with NDM-type enzymes, was reported in a $P$. aeruginosa isolate from Italy [221]. The bla $a_{\mathrm{FIM}-1}$ gene was chromosomally located and was associated with ISCR19-like elements that were likely involved in its capture and mobilisation [221]; its origin remains unknown.

\subsection{Class $C \beta$-lactamases}

\subsubsection{Acinetobacter baumannii}

Acinetobacter baumannii naturally produces a gene encoding an AmpC-type cephalosporinase. This gene is usually expressed at a basal and low level, therefore the amount of AmpC produced does not have a significant impact on the activity of expanded-spectrum cephalosporins [222]. The presence of a specific IS element ISAba1 (belonging to the IS4 family) upstream of this naturally occurring ampC gene provides promoter sequences enhancing its expression, resulting in resistance to broad-spectrum cephalosporins (but sparing carbapenems) [223]. By studying a series of $A$. baumannii strains, a variety of $A m p C$ variants may be identified and these variants have been named ADC-type (Acinetobacterderived cephalosporinase) enzymes [224]. Some ADC variants, such as ADC-33 and ADC-56, possess a slight extended activity towards expanded-spectrum cephalosporins, which allows the classification of these enzymes as extended-spectrum AmpC (ESAC) $[225,226]$. Indeed, they do hydrolyse ceftazidime more efficiently, and in addition they hydrolyse fourth-generation cephalosporins such as cefepime, whereas wild-type AmpC enzymes do not. The true clinical significance of these enzymes remains unknown. No acquired AmpC-type-encoding gene has been identified so far in $A$. baumannii.

\subsubsection{Pseudomonas aeruginosa}

A chromosomal gene encoding an AmpC-type cephalosporinase is also intrinsic to $P$. aeruginosa. This ampC gene is associated with a LysR-type regulatory gene with which some $\beta$-lactam molecules may interact, leading to overexpression of this ampC gene [227]. Some $\beta$-lactams such as carbapenems are inducers of $a m p C$ gene expression, although they are not substrates of these cephalosporinases. Selection of mutants overproducing the frequently observed in $P$. aeruginosa, leading to acquired resistance to ticarcillin, piperacillin and broad-spectrum cephalosporins (ceftazidime) [227]. In addition, insertion of the IS1669 element into the LysR regulatory gene (also known as the $a m p R$ gene) of $a m p C$ may lead to the overexpression of this enzyme [228]. Apart from these mechanisms leading to increased resistance to expanded-spectrum cephalosporins, very peculiar AmpC-type enzymes of $P$. aeruginosa have been identified possessing a broadened hydrolytic activity towards imipenem [229]. They correspond to naturally occurring, chromosomally encoded AmpC-type $\beta$-lactamases possessing an alanine residue at position 105 conferring an additional weak carbapenemase activity $[229,230]$. The true clinical significance of these enzymes as a source of carbapenem resistance remains to be clarified.

\subsection{Class $D \beta$-lactamases}

Class D $\beta$-lactamases, also known as oxacillinases, are $\beta$ lactamases grouped in a heterogeneous class of enzymes either with respect to their structural or biochemical properties [231]. These enzymes all hydrolyse amoxicillin and cefalotin and their 
Table 4

Ambler class D $\beta$-lactamases known in Acinetobacter baumannii and Pseudomonas aeruginosa.

\begin{tabular}{|c|c|c|c|c|}
\hline Host & Enzyme subfamily & Additional OXA members & Phenotype & Reference(s) \\
\hline \multirow{5}{*}{$\begin{array}{l}\text { Acinetobacter } \\
\text { baumannii }\end{array}$} & OXA-23 (ARI-1) & OXA-27, OXA-49 & CHDL & {$[107,236,237]$} \\
\hline & OXA-40 & OXA-25, OXA-26, OXA-72 & CHDL & {$[232,238-245]$} \\
\hline & OXA-58 & OXA-96, OXA-97 & CHDL & {$[117,246-248]$} \\
\hline & OXA-143 & & CHDL & [249] \\
\hline & OXA-235 & OXA-236, OXA-237 & CHDL & [250] \\
\hline \multirow{8}{*}{$\begin{array}{l}\text { Pseudomonas } \\
\text { aeruginosa }\end{array}$} & OXA-2 & OXA-15, OXA-32, OXA-34, OXA-36, OXA-141, OXA-161 & ES-OXA & {$[231,252-254]$} \\
\hline & OXA-10 & $\begin{array}{l}\text { OXA-11, OXA-13, OXA-14, OXA-16, OXA-17, OXA-19, OXA-28, OXA-129, OXA-142, } \\
\text { OXA-145, OXA-147, OXA-183 }\end{array}$ & ES-OXA & {$[231,255-262]$} \\
\hline & OXA-1 & OXA-31 & ES-OXA & [263] \\
\hline & OXA-56 & OXA-128 & ES-OXA & [261] \\
\hline & OXA-18 & & ES-OXA & [264] \\
\hline & OXA-45 & & ES-OXA & [265] \\
\hline & OXA-40 & & CHDL & [267] \\
\hline & OXA-198 & & CHDL & [268] \\
\hline
\end{tabular}

CHDL, carbapenem-hydrolysing class D $\beta$-lactamase; ES-OXA, extended-spectrum oxacillinase.

activities are usually not significantly inhibited by clavulanic acid [232]. Some class D $\beta$-lactamases hydrolyse expanded-spectrum cephalosporins and a few have been identified in $P$. aeruginosa, but none in $A$. baumannii. Most of these broad-spectrum class $D$ $\beta$-lactamases, also called ES-OXA, are point-mutant derivatives of narrow-spectrum $\beta$-lactamases [231]. In contrast, carbapenemase activity is also an intrinsic property of many class D $\beta$-lactamases, therefore terming them carbapenem-hydrolysing class D $\beta$-lactamases (CHDLs) [231]

\subsubsection{Acinetobacter baumannii}

Acinetobacter baumannii possesses naturally occurring class $\mathrm{D} \beta$ lactamases, known as OXA-51-like enzymes [233]. These enzymes exhibit weak carbapenemase activity and are classified as CHDLs. Noticeably, the corresponding genes are not (or only weakly) expressed in most isolates. However, once overexpressed they may subsequently be involved in reduced susceptibility to carbapenems [234]. Overexpression of these genes encoding OXA-51-like enzymes is often driven by the insertion of an ISAba1 element upstream of the bla $a_{\mathrm{OXA}-51}$-like gene, providing strong promoter sequences.

In addition to these naturally occurring class $\mathrm{D} \beta$-lactamases, several acquired class $D \beta$-lactamases have been identified as a source of carbapenem resistance in A. baumannii [107]. These CHDLs confer only reduced susceptibility to carbapenems, but they spare broad-spectrum cephalosporins. Therefore, the high resistance to carbapenems often observed in many A. baumannii strains results from the association between a CHDL and other resistance mechanisms, including porin loss and overexpression of efflux systems [107]. Five main groups of acquired CHDLs have been described in A. baumannii, corresponding to OXA-23-, OXA-40-, OXA-58-, OXA-143 and OXA-235-like enzymes. The first and most common subgroup of CHDLs is made of OXA-23, OXA-27 and OXA49 (Table 4) [107]. The bla $a_{\text {OXA-23-like genes are chromosome- or }}$ plasmid-encoded and they are part of transposons, namely $\operatorname{Tn} 2006$ and Tn2007 [235]. OXA-23-like enzymes are the most widespread CHDLs in A. baumannii worldwide and they have been identified on all continents [107,236]. OXA-23-producing A. baumannii are the most common sources of nosocomial outbreaks with carbapenemresistant $A$. baumannii $[231,237]$. A second group of acquired CHDLs in A. baumannii comprises OXA-25, OXA-26, OXA-40 (formerly known as OXA-24) and OXA-72 (Table 4). OXA-25 has been identified in carbapenem-resistant $A$. baumannii isolates recovered from Spain, and OXA-26 in Belgium [238]. The OXA-40 CHDL was originally identified in a carbapenem-resistant $A$. baumannii isolate in France recovered from a Portuguese patient [239], and then extensively identified in Portugal and Spain, as in other parts of the world $[231,240,241]$. The bla $a_{\mathrm{OXA}-40}$-like genes may be either chromosome- or plasmid-located. OXA-72 has also been identified in different parts of the world (Brazil, Lithuania, Croatia), but predominantly in Asia (China, South Korea, Taiwan, Japan) [231,242-245]. A third group of CHDLs corresponds to OXA-58 and its structurally related enzymes (OXA-96 and OXA-97), first identified from a carbapenem-resistant $A$. baumannii isolate recovered in France [246] in the context of a nosocomial outbreak in a burn unit [247]. This bla $a_{\text {OXA-58 }}$ gene has now been reported worldwide [231], being always plasmid-borne and associated with IS elements at the origin of its expression [107]. OXA-96 and OXA-97 are point-mutant variants of OXA-58 sharing the same hydrolytic properties and identified in Singapore and Tunisia, respectively $[117,248]$

The OXA-143 CHDL was identified in 2009 from a clinical A. baumannii isolate that had been recovered in Brazil [249]. It shares $88 \%$ amino acid identity with OXA-40, 63\% with OXA-23 and 52\% with OXA-58. Its substrate profile was similar to those of other CHDLs and its corresponding gene was not integron- or transposonencoded [249].

Ultimately, a novel subclass of CHDLs has recently been reported from isolates recovered in the USA and Mexico. This subgroup includes OXA-235, OXA-236 and OXA-237 [250], and the corresponding genes have been identified either on chromosomes or plasmids, and bracketed by two copies of ISAba1 [250].

\subsubsection{Pseudomonas aeruginosa}

Pseudomonas aeruginosa produces a naturally occurring class D $\beta$-lactamase, OXA-50, that does not contribute to the overall $\beta$-lactam resistance pattern of $P$. aeruginosa, except for latamoxef [251]. Most of the class D $\beta$-lactamases able to hydrolyse expanded-spectrum cephalosporins have been identified in $P$. aeruginosa. There are two main types of expanded-spectrum class D $\beta$-lactamases (ES-OXAs). Some are point-mutant derivatives of narrow-spectrum class $D \beta$-lactamases, with amino acid substitutions enlarging their spectrum of hydrolysis towards expanded-spectrum cephalosporins. These ES-OXAs mainly derive from the narrow-spectrum $\beta$-lactamases OXA-10 (OXA-11, -13, $-14,-16,-17,-19,-28,-129,-142,-145,-147$ and -183$)$, OXA2 (OXA-15, -32, -34, -36, -141 and -161) and OXA-1 (OXA-31) (Table 4) [231,252-263]. Other ES-OXAs share only weak amino acid identity with these latter enzymes. OXA-18, which is inhibited by clavulanic acid, was the first identified ES-OXA in a $P$. aeruginosa isolate in Paris from a patient previously hospitalised in Sicily [264]. This enzyme shares $<50 \%$ amino acid identity with the other class D $\beta$-lactamases. OXA-45 is another ES-OXA, identified from a multidrug-resistant Texan $P$. aeruginosa isolate co-expressing 
the class B $\beta$-lactamase VIM-7 [265]. OXA-45 shares the highest identity with OXA-18 (66\%) and, as for OXA-18, its activity is well inhibited by clavulanic acid. Interestingly, the $b l a_{\text {OXA-45 }}$ gene, located on a 24-kb plasmid, was not found as a form of a gene cassette but associated with two copies of an ISCR5-like element [266].

Only two acquired class $\mathrm{D} \beta$-lactamases compromising the efficacy of carbapenems have been reported from $P$. aeruginosa. One is OXA-40, known to be widespread in A. baumannii, which was reported in a P. aeruginosa isolate in Spain in 2006 [267]. The other is OXA-198 characterised in a P. aeruginosa isolate from Belgium [268], sharing $<30 \%$ amino acid identity with other CHDLs reported in Gram-negative organisms. The bla OXA-198 gene was harboured by a class 1 integron carried by a non-typeable ca. $46-\mathrm{kb}$ plasmid [268].

\section{Broad resistance to aminoglycosides}

Aminoglycosides are used in the treatment of a broad range of life-threatening infections. The activity of aminoglycosides depends on binding to a highly conserved motif of $16 \mathrm{~S}$ rRNA. Mechanisms of aminoglycosides resistance include decreased outer membrane permeability, active efflux and amino acid substitutions in ribosomal proteins, whereas the most common resistance mechanism is enzymatic leading to modification of the drug. Methylation of $16 \mathrm{~S}$ ribosomal RNA has recently been demonstrated to be another mechanism of resistance encountered in Gram-negative organisms, corresponding to a modification of the antibiotic target [269]. Methylases actually interfere in the binding of these antibiotics to their site of action. These 16S rRNA methylases confer a high level of resistance to clinically useful aminoglycosides such as amikacin, gentamicin and tobramycin [269,270]. The corresponding genes are associated with transposon structures, which are themselves located on transferable plasmids, enhancing their horizontal spread. Isolates producing 16S rRNA methylases are multidrug-resistant, in particular to broad-spectrum $\beta$-lactams through the production of ESBLs or MBLs. This multidrug resistance pattern makes treatment of these infections particularly challenging. Ten 16S rRNA methylases have been identified among Gram-negative isolates, namely ArmA, RmtA, RmtB, RmtC, RmtD, RmtD2, RmtE, RmtF, RmtG and NpmA [270-273]. The origin of these genes is likely Streptomyces [271] and their prevalence remains unknown depending on the geographical location (possibly more frequent in Asia).

\subsection{Acinetobacter baumannii}

The ArmA enzyme has been reported in many A. baumannii worldwide, conferring high-level resistance to all aminoglycosides. Such isolates have been identified in China [274], South Korea [275,276], Vietnam [277], Japan [278], North America [279], Norway [280], Italy [281], Bulgaria [282], Iran [283] and Algeria [136]. The armA gene was always found to be located on a functional composite transposon Tn1548 [279]. Despite being quite widespread among $A$. baumannii strains, the $\operatorname{arm} A$ gene possesses a GC content of $30 \%$, which significantly differs from that of the $A$. baumannii core genome estimated at ca. $39 \%$. This highlights the fact that this gene was acquired horizontally from a source that still remains unknown [279]. Noteworthy, the ArmA-encoding gene is often identified among OXA-23-producing A. baumannii strains, however both resistance genes are not physically linked on a single plasmid [280-282].

Apart from numerous reports of ArmA-producing A. baumannii isolates, the $16 \mathrm{~S}$ rRNA methylase RmtB has recently been identified in nine A. baumannii isolates from Vietnam [277].

\subsection{Pseudomonas aeruginosa}

The first 16S rRNA methylase recovered in $P$. aeruginosa was identified in 2003. It was a clinical isolate from Japan producing RmtA [284,285]. Other RmtA-producing P. aeruginosa were then identified in South Korea in 2009 [286]. The rmtA gene is located on mobile genetic elements such as transposon Tn5041 [285].

In 2007, the RmtD methylase was firstly reported from a pandrug-resistant $P$. aeruginosa clinical isolate co-producing the MBL SPM-1 in Brazil [287]. RmtD shares 40\% amino acid identity with RmtA, and the genetic structures surrounding both corresponding genes shared similar features [287]. Subsequently, another study underscored that co-production of the MBL SPM-1 and the $16 \mathrm{~S}$ rRNA methylase RmtD was common among imipenemresistant $P$. aeruginosa isolates recovered in hospitals in São Paulo, Brazil [288]. In these isolates, both the bla $a_{\mathrm{SPM}-1}$ and $r m t D$ genes were found to be chromosomally located. The ArmA enzyme was also identified in P. aeruginosa co-producing the MBL IMP-1 in South Korea [289].

\section{Broad resistance to fluoroquinolones}

In Gram-negative organisms, acquisition of resistance to quinolones may be related to chromosomal mutations in genes encoding the topoisomerases or to mutations in the efflux pump regulation systems. In addition, plasmid-mediated quinolone resistance genes (coding for the Qnr proteins) have been identified in Enterobacteriaceae. These acquired Qnr proteins have not been identified in non-fermenters. In $P$. aeruginosa and $A$. baumannii, a single mutation in the gyrA gene encoding DNA gyrase is sufficient to confer clinically high-level resistance levels to fluoroquinolones. This is due to the fact that these species intrinsically possess a decreased susceptibility to these antibiotics owing to low permeability or constitutive expression of efflux pumps.

\subsection{Acinetobacter baumannii}

Overexpression of efflux pumps is a source of acquired resistance to fluoroquinolones in this species. Involvement of the ade $A B C$ operon encoding the AdeA, AdeB and AdeC proteins forming a resistance-nodulation-cell division (RND) efflux system has been demonstrated [290]. In this case, not only fluoroquinolones but also aminoglycosides, tetracyclines, chloramphenicol and trimethoprim are substrates of this efflux system. Therefore, co-selection of this kind of mechanism with non-quinolone antibiotic molecules is possible. Similarly, overexpression of the adeIJK operon encoding another RND efflux system of $A$. baumannii has also been shown to interfere with different antibiotics, including the fluoroquinolones [291]. Finally, overproduction of the AbeM efflux system [belonging to the multi-antimicrobial extrusion protein (MATE) family] also contributes to acquired resistance to fluoroquinolones [292].

\subsection{Pseudomonas aeruginosa}

As shown in $A$. baumannii, acquired resistance to fluoroquinolones, apart from being related to mutations in topoisomerase-encoding genes, is mainly related to efflux systems. Their downregulation or upregulation (depending on whether the regulator is positive or negative) contribute significantly to reduced activity of fluoroquinolones. In $P$. aeruginosa, involvement of the MexAB-OprM RND-type system, which expression is constitutive, has been demonstrated, and its wide effect has been highlighted, conferring reduced susceptibility also to chloramphenicol, tetracyclines and $\beta$-lactams [293]. Likewise, the mexCD-oprJ operon confers extrusion ability with regard to quinolones, penicillins and tetracyclines. Nevertheless, this impact is seen only when the 
negative regulatory protein $\mathrm{NfxB}$ is altered, indicating that constitutive expression of this efflux system does not contribute to this resistance trait [294]. Another efflux system is MexEF-OprN, which is also not involved in reduced susceptibility unless overexpression is observed [295].

\section{Resistance to tigecycline}

Tigecycline, a semisynthetic derivative of minocycline, has a peculiar mechanism of action and overcomes the widely distributed tet gene-encoded resistance mechanism known to confer resistance to tetracycline. Tigecycline shows good activity towards Gram-negative pathogens that may produce a large array of resistance mechanisms, including ESBLs and carbapenemases [296]. The activity of tigecycline against $A$. baumannii is overall good, and successful results have been reported clinically [296]. Resistance has been noted on several occasions and might be due to upregulation of the AdeABC multidrug efflux pump [297]. Another A. baumannii-specific efflux system, named AdeIJK, has also been shown to interfere with the efficacy of tigecycline, acting synergistically with AdeABC [291]. Despite the fact that tigecycline has been shown to also be a substrate of the AdeFGH efflux pump [298], only AdeABC and AdeIJK efflux pumps appear to be involved in its resistance in clinical isolates [298-300]. Noteworthy, tigecycline resistance levels in $A$. baumannii isolates may increase during therapy with tigecycline in the case of brief exposure to the drug, compromising its efficiency [301]. It was recently reported that a mutation in the trm gene, encoding a methyltransferase, was associated with decreased susceptibility to tigecycline in an A. baumannii strain [302]. This Trm enzyme could therefore play a role in resistance to tigecycline, however further studies are needed to clarify the possible role of this methyltransferase in the decreased susceptibility to tigecycline [302].

\section{Resistance to colistin}

During the past decade, we have witnessed a renewal of clinical interest in polymyxins (colistin) owing to two concomitant facts: (i) the emergence of carbapenem-, cephalosporinand aminoglycoside-resistant Gram-negative isolates; and (ii) the paucity of novel marketed antibiotic molecules. Actually, polymyxins remain most often active against these multidrug-resistant isolates [303]. Emergence of colistin resistance in relation to increased usage is worrisome since polymyxins are the last remaining therapeutic option in many cases. Acquisition of resistance is mostly related to modifications of the lipopolysaccharide (LPS) biosynthesis pathway.

\subsection{Acinetobacter baumannii}

Two mechanisms of resistance to colistin have been described in A. baumannii: (i) alterations of the lipid A component of LPS resulting from mutations in the PmrAB two-component system [304,305]; and (ii) complete loss of LPS production resulting from mutations in the $\operatorname{lp} x A, \operatorname{lp} x C$ and $\operatorname{lp} x D$ genes encoding the enzymes that catalyse the first steps in LPS biosynthesis [306]. Resistance to colistin in A. baumannii clinical isolates is rarely reported, however evaluation of colistin susceptibility is difficult and often inaccurate, considering that many laboratories do not use the gold standard technique for testing, which is microdilution [307]. The first report of colistin-resistant A. baumannii was from South Korea in 2005 [303]. High colistin resistance rates were then reported in other Korean hospitals in 2007 [308], and an outbreak of pandrugresistant (including colistin) A. baumannii strains was reported in Spain in 2009 [309]. More recently, colistin-resistant A. baumannii were recovered in Iran and the USA [310,311]. Noteworthy, development of heteroresistance to colistin in an in vitro model might compromise the use of colistin as a therapeutic option [312].

\subsection{Pseudomonas aeruginosa}

The emergence of colistin-resistant $P$. aeruginosa isolates has also been reported worldwide [313-315]. As observed in A. baumannii, resistance to polymyxins is associated with modifications of the lipid A component of LPS. Several two-component regulatory systems, such as PmrAB, PhoPQ, ParRS, CprRS and ColRS, are involved in resistance to polymyxins in P. aeruginosa [316-318]. A recent study identified nine genes with amino acid alterations and altered expression levels in colistin-resistant $P$. aeruginosa isolates compared with an isogenic colistin-susceptible isolate [319]. Thus, resistance to colistin is basically mediated by a complicated regulatory network involving a large array of chromosomal genes, which is currently under investigation worldwide, with some side experiments aiming to evaluate the fitness cost of such resistance.

\section{Concluding remarks}

Increasing rates of bacterial resistance among non-fermenters are threatening the effectiveness of antibiotics used as last-resort therapeutic options. In A. baumannii and P. aeruginosa, acquisition of resistance traits to these molecules is becoming more and more frequent, leading to multidrug and pandrug resistance. The last years have shown that: (i) most of the broad-spectrum resistance patterns identified in Enterobacteriaceae may be also identified in $P$. aeruginosa and A. baumannii; (ii) accumulation of unrelated resistance mechanisms (e.g. to $\beta$-lactams and aminoglycosides) is observed daily worldwide; and (iii) A. baumannii, although being a weak pathogen compared with $P$. aeruginosa, may play a significant role in spreading broad-spectrum resistance genes to other Gram-negative organisms. International travel and transfer of hospitalised patients further enhances this spread. In parallel, antibiotic selective pressure is also on the rise, in particular for these last-resort antibiotics that have been used only scarcely until recently. Since very few novel and effective antibiotics for the treatment of infections due to multidrug-resistant Gram-negatives isolates are going to be launched in a near future, there is an urgent need to implement strategies that may slow the development of acquired resistance. Use of rapid diagnostic techniques for detection of resistance traits, development of selective media for early recognition of colonised patients, and improvement of antibiotic stewardship may contribute to this containment strategy against antibiotic resistance.

Funding: This work was funded by the University of Fribourg (Fribourg, Switzerland).

Competing interests: None declared.

Ethical approval: Not required.

\section{References}

[1] Weldhagen GF, Poirel L, Nordmann P. Ambler class A extended-spectrum $\beta$-lactamases in Pseudomonas aeruginosa: novel developments and clinical impact. Antimicrob Agents Chemother 2003;47:2385-92.

[2] Kolayli F, Gacar G, Karadenizli A, Sanic A, Vahaboglu H. PER-1 is still widespread in Turkish hospitals among Pseudomonas aeruginosa and Acinetobacter spp. FEMS Microbiol Lett 2005:15:241-5.

[3] Yong D, Shin H, Kim S, Lim Y, Yum JH, Lee K, et al. High prevalence of PER1 extended-spectrum $\beta$-lactamase-producing Acinetobacter spp. in Korea. Antimicrob Agents Chemother 2003;47:1749-51. 
[4] Szabó D, Szentandrássy J, Juhász Z, Katona K, Nagy K, Rókusz L. Imported PER-1 producing Pseudomonas aeruginosa, PER-1 producing Acinetobacter baumani and VIM-2-producing Pseudomonas aeruginosa strains in Hungary. Ann Clin Microbiol Antimicrob 2008; 7:12.

[5] Naas T, Nordmann P, Heidt A. Intercountry transfer or PER-1 extendedspectrum $\beta$-lactamase-producing Acinetobacter baumannii from Romania. Int Antimicrob Agents 2007:29:226-8.

[6] Naas T, Kernbaum S, Allali S, Nordmann P. Multidrug-resistant Acinetobacter baumannii, Russia. Emerg Infect Dis 2007;13:669-71.

[7] Naas T, Bogaerts P, Bauraing C, Degheldre Y, Glupczynski Y, Nordmann P. Emergence of PER and VEB extended-spectrum $\beta$-lactamases in Acinetobacter baumannii in Belgium. J Antimicrob Chemother 2006;58:178-82.

[8] Hujer KM, Hujer AM, Hulten EA, Bajaksouzian S, Adams JM, Donskey CJ, et al. Analysis of antibiotic resistance genes in multidrug-resistant Acinetobacter sp. isolates from military and civilian patients treated at the Walter Reed Army Medical Center. Antimicrob Agents Chemother 2006;50:4114-23.

[9] Strateva T, Todorova A, Ouzounova-Raykova V, Mitov I. Emergence of a PER-1 extended-spectrum $\beta$-lactamase-producing Acinetobacter baumannii clinical isolate in Bulgaria. J Chemother 2008;20:391-2.

[10] Litake GM, Ghole VS, Niphadkar KB, Joshi SG. PER-1-type extended-spectrum $\beta$-lactamase-producing Acinetobacter baumannii clinical isolates from India. Int J Antimicrob Agents 2009;34:388-9.

[11] Zhang JP, Zhu W, Tian SF, Chu YZ, Chen BY. Molecular characteristics and resistant mechanisms of imipenem-resistant Acinetobacter baumannii isolates in Shenyang, China. J Microbiol 2010;48:689-94.

[12] Farajnia S, Azhari F, Alikhani MY, Hosseini MK, Peymani A, Sohrabi N. Prevalence of PER and VEB type extended spectrum $\beta$-lactamases among multidrug resistant Acinetobacter baumannii isolates in North-West of Iran. Iran J Basic Med Sci 2013;16:751-5.

[13] Opazo A, Vali L, Al Obaid K, Dashti AA, Amyes SG. Novel genetic structure

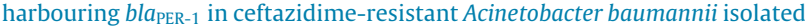
from Kuwait. Int J Antimicrob Agents 2014:43:383-4.

[14] Poirel L, Cabanne L, Vahaboglu H, Nordmann P. Genetic environment and expression of the extended-spectrum $\beta$-lactamase bla $a_{\mathrm{PER}-1}$ gene in Gramnegative bacteria. Antimicrob Agents Chemother 2005;49:1708-13.

[15] Pasterán F, Rapoport M, Petroni A, Faccone D, Corso A, Galas M, et al. Emergence of PER-2 and VEB-1a in Acinetobacter baumannii strains in the Americas. Antimicrob Agents Chemother 2006;50:3222-4.

[16] Al-Hassan L, El Mahallawy H, Amyes SG. First report of bla PER-3 $_{\text {in Acinetobacter }}$ baumannii. Int J Antimicrob Agents 2013:41:93-4.

[17] Bonnin RA, Potron A, Poirel L, Lécuyer H, Néri R, Nordmann PPER-7. an extended-spectrum $\beta$-lactamase with increased activity toward broadspectrum cephalosporins in Acinetobacter baumannii. Antimicrob Agents Chemother 2011;55:2424-7.

[18] Opazo A, Sonnevend A, Lopes B, Hamouda A, Ghazawi A, Pal T, et al. Plasmid-encoded PER-7 $\beta$-lactamase responsible for ceftazidime resistance in Acinetobacter baumannii isolated in the United Arab Emirates. J Antimicrob Chemother 2012;67:1619-22.

[19] Poirel L, Naas T, Guibert M, Chaibi EB, Labia R, Nordmann P. Molecular and biochemical characterization of VEB-1, a novel class A extended-spectrum B-lactamase encoded by an Escherichia coli integron gene. Antimicrob Agents Chemother 1999;43:573-81.

[20] Poirel L, Menuteau O, Agoli N, Cattoen C, Nordmann P. Outbreak of extendedspectrum $\beta$-lactamase VEB-1-producing isolates of Acinetobacter baumanni in a French hospital. J Clin Microbiol 2003:41:3542-7.

[21] Naas T, Coignard B, Carbonne A, Blanckaert K, Bajolet O, Bernet C, et al. VEB-1 extended-spectrum $\beta$-lactamase-producing Acinetobacter baumannii, France. Emerg Infect Dis 2006;12:1214-22.

[22] Poirel L, Mugnier PD, Toleman MA, Walsh TR, Rapoport MJ, Petroni A et al. ISCR2, another vehicle for bla $a_{\mathrm{VFB}}$ gene acquisition. Antimicrob Agents Chemother 2009;53:4940-3.

[23] Huang LY, Chen TL, Lu PL, Tsai CA, Cho WL, Chang FY, et al. Dissemination of multidrug-resistant, class 1 integron-carrying Acinetobacter baumannii isolates in Taiwan. Clin Microbiol Infect 2008;14:1010-9.

[24] Poirel L, Le Thomas I, Naas T, Karim A, Nordmann P. Biochemical sequence analyses of GES-1, a novel class A extended-spectrum $\beta$-lactamase, and the class 1 integron $\ln 52$ from Klebsiella pneumoniae. Antimicrob Agent Chemother 2000;44:622-32.

[25] Moubareck C, Brémont S, Conroy MC, Courvalin P, Lambert T. GES-11, a novel integron-associated GES variant in Acinetobacter baumannii. Antimicrob Agents Chemother 2009;53:3579-81.

[26] Bogaerts P, Naas T, El Garch F, Cuzon G, Deplano A, Delaire T, et al GES extended-spectrum $\beta$-lactamases in Acinetobacter baumannii isolates in Belgium. Antimicrob Agents Chemother 2010;54:4872-8.

[27] Karah N, Giske CG, Sundsfjord A, Samuelsen O. A diversity of OXAcarbapenemases and class 1 integrons among carbapenem-resistant Acinetobacter baumannii clinical isolates from Sweden belonging to different international clonal lineages. Microb Drug Resist 2011;17:545-9.

[28] Bonnin RA, Rotimi VO, Al Hubail M, Gasiorowski E, Al Sweih N, Nordmann $\mathrm{P}$, et al. Wide dissemination of GES-type carbapenemases in Acinetobacter baumannii isolates in Kuwait. Antimicrob Agents Chemother 2013;57:183-8.

[29] Cicek AC, Saral A, Iraz M, Ceylan A, Duzgun AO, Peleg AY, et al. OXA- and GES type $\beta$-lactamases predominate in extensively drug-resistant Acinetobacter baumannii isolates from a Turkish university hospital. Clin Microbiol Infect 2014;20:410-5.
[30] Charfi-Kessis K, Mansour W, Ben Haj Khalifa A, Mastouri M, Nordmann P, Aouni M, et al. Multidrug-resistant Acinetobacter baumannii strains carrying the bla $a_{\mathrm{OXA}-23}$ and the $b l a_{\mathrm{GES}-11}$ genes in a neonatology center in Tunisia. Microb Pathog 2014;74:20-4.

[31] Delbrück H, Bogaerts P, Kupper MB, Rezende de Castro R, Bennink S, Glupczynski Y, et al. Kinetic and crystallographic studies of extended-spectrum GES-11, GES-12, and GES-14 $\beta$-lactamases. Antimicrob Agents Chemother 2012;56:5618-25.

[32] Castanheira M, Costello SE, Woosley LN, Deshpande LM, Davies TA, Jones RN. Evaluation of clonality and carbapenem resistance mechanisms among Acinetobacter baumannii-Acinetobacter calcoaceticus complex and Enterobacteriaceae isolates collected in European and Mediterranean countries and detection of two novel $\beta$-lactamases, GES-22 and VIM-35. Antimicrob Agents Chemother 2014;58:7358-66.

[33] Naas T, Namdari F, Réglier-Poupet H, Poyart C, Nordmann P. Panresistant extended-spectrum $\beta$-lactamase SHV-5-producing Acinetobacter baumannii from New York City. J Antimicrob Chemother 2007;60:1174-6.

[34] Endimiani A, Luzzaro F, Migliavacca R, Mantengoli E, Hujer AM, Hujer KM, et al. Spread in an Italian hospital of a clonal Acinetobacter baumannii strain producing the TEM-92 extended-spectrum $\beta$-lactamase. Antimicrob Agents Chemother 2007;51:2211-4

[35] Naiemi NA, Duim B, Savelkoul PH, Spanjaard L, de Jonge E, Bart A, et al. Widespread transfer of resistance genes between bacterial species in an intensive care unit: implications for hospital epidemiology. J Clin Microbiol 2005;43:4862-4.

[36] Nagano N, Nagano Y, Cordevant C, Shibata N, Arakawa Y. Nosocomial transmission of CTX-M-2 $\beta$-lactamase-producing Acinetobacter baumannii in a neurosurgery ward. J Clin Microbiol 2004;42:3978-84.

[37] Adams-Haduch JM, Paterson DL, Sidjabat HE, Pasculle AW, Potoski BA, Muto $\mathrm{CA}$, et al. Genetic basis of multidrug resistance in Acinetobacter baumannii clinical isolates at a tertiary medical center in Pennsylvania. Antimicrob Agents Chemother 2008;52:3837-43.

[38] Celenza G, Pellegrini C, Caccamo M, Segatore B, Amicosante G, Perilli M. Spread of bla $a_{\text {CTX-M-type }}$ and bla $a_{\text {PER-2 }} \beta$-lactamase genes in clinical isolates from Bolivian hospitals. J Antimicrob Chemother 2006;57:975-8.

[39] Shakil S, Khan AU. Detection of CTX-M-15-producing and carbapenemresistant Acinetobacter baumannii strains from urine from an Indian hospital. J Chemother 2010;22:324-7.

[40] Potron A, Munoz-Price LS, Nordmann P, Cleary T, Poirel L. Genetic features of CTX-M-15-producing Acinetobacter baumannii from Haiti. Antimicrob Agents Chemother 2011;55:5946-8.

[41] Potron A, Poirel L, Croizé J, Chanteperdrix V, Nordmann P. Genetic and biochemical characterization of the first extended-spectrum CARB-type B-lactamase, RTG-4, from Acinetobacter baumannii. Antimicrob Agents Chemother 2009;53:3010-6.

[42] Nordmann P, Ronco E, Naas T, Duport C, Michel-Briand Y, Labia R. Characterization of a novel extended-spectrum $\beta$-lactamase from Pseudomonas aeruginosa. Antimicrob Agents Chemother 1993;37:962-9.

[43] Claeys G, Verschraegen G, de Baere T, Vaneechoutte M. PER-1 $\beta$-lactamaseproducing Pseudomonas aeruginosa in an intensive care unit. J Antimicrob Chemother 2000;45:924-5.

[44] Luzzaro F, Mantengoli E, Perilli M, Lombardi G, Orlandi V, Orsatti A, et al. Dynamics of a nosocomial outbreak of multidrug-resistant Pseudomonas aeruginosa producing the PER-1 extended-spectrum $\beta$-lactamase. J Clin Microbiol 2001:39:1865-70.

[45] Tato M, Valverde A, Coque TM, Cantón R. PER-1 multiresistant Pseudomonas aeruginosa strain in Spain [in Spanish]. Enferm Infecc Microbiol Clin 2006;24:472-3.

[46] Empel J, Filczak K, Mrówka A, Hryniewicz W, Livermore DM, Gniadkowski M. Outbreak of Pseudomonas aeruginosa infections with PER-1 extended-spectrum $\beta$-lactamase in Warsaw, Poland: further evidence for an international clonal complex. J Clin Microbiol 2007;45:2829-34.

[47] Libisch B, Poirel L, Lepsanovic Z, Mirovic V, Balogh B, Paszti J, et al. Identification of PER-1 extended-spectrum $\beta$-lactamase producing Pseudomonas aeruginosa clinical isolates of the international clonal complex CC11 from Hungary and Serbia. FEMS Immunol Med Microbiol 2008;54:330-8.

[48] Ktari S, Mnif B, Znazen A, Rekik M, Mezghani S, Mahjoubi-Rhimi F, et al. Diversity of $\beta$-lactamases in Pseudomonas aeruginosa isolates producing metallo- $\beta$-lactamase in two Tunisian hospitals. Microb Drug Resist 2011;17:25-30.

[49] Yamano Y, Nishikawa T, Fujimura T, Yutsudou T, Tsuji M, Miwa H. Occurrence of PER-1 producing clinical isolates of Pseudomonas aeruginosa in Japan and their susceptibility to doripenem. J Antibiot (Tokyo) 2006;59:791-6.

[50] Qing Y, Cao KY, Fang ZL, Huang YM, Zhang XF, Tian GB, et al. Outbreak of PER-1 and diversity of $\beta$-lactamases among ceftazidime-resistant Pseudomonas aeruginosa clinical isolates in China. J Med Microbiol 2014;63: 386-92.

[51] Ranellou K, Kadlec K, Poulou A, Voulgaru E, Vrioni G, Schwarz S, et al. Detection of Pseudomonas aeruginosa isolates of the international clonal complex 11 carrying the bla $a_{\text {PER-1 }}$ extended-spectrum $\beta$-lactamase gene in Greece. J Antimicrob Chemother 2012;67:357-61.

[52] Mirsalehian A, Feizabadi M, Nakhjavani FA, Jabalameli F, Goli H, Kalantari N. Detection of VEB-1, OXA-10 and PER-1 genotypes in extended-spectrum $\beta$-lactamase-producing Pseudomonas aeruginosa strains isolated from burn patients. Burns 2009;36:70-4. 
[53] Naas T, Poirel L, Karim A, Nordmann P. Molecular characterization of In50, a class 1 integron encoding the gene for the extended-spectrum $\beta$ lactamase VEB-1 in Pseudomonas aeruginosa. FEMS Microbiol Lett 1999;176: 411-9.

[54] Girlich D, Naas T, Leelaporn A, Poirel L, Fennewald M, Nordmann P. Nosocomial spread of the integron-located VEB-1-like cassette encoding an extended-spectrum $\beta$-lactamase in Pseudomonas aeruginosa in Thailand. Clin Infect Dis 2002:34:603-11.

[55] Poirel L, Rotimi VO, Mokaddas EM, Karim A, Nordmann P. VEB-1-like extended-spectrum $\beta$-lactamases in Pseudomonas aeruginosa, Kuwait. Emerg Infect Dis 2001;7:468-70.

[56] Aubert D, Girlich D, Naas T, Nagarajan S, Nordmann P. Functional and structural characterization of the genetic environment of an extended-spectrum $\beta$-lactamase bla $a_{\mathrm{VEB}}$ gene from a Pseudomonas aeruginosa isolate obtained in India. Antimicrob Agents Chemother 2004;48:3284-90.

[57] Strateva T, Ouzounova-Raykova V, Markova B, Todorova A, Marteva-Proevska Y, Mitov I. Widespread detection of VEB-1-type extended-spectrum $\beta$ lactamases among nosocomial ceftazidime-resistant Pseudomonas aeruginosa isolates in Sofia, Bulgaria. J Chemother 2007;19:140-5.

[58] Woodford N, Zhang J, Kaufmann ME, Yarde S, Tomas Mdel M, Faris C, et al. Detection of Pseudomonas aeruginosa isolates producing VEB-type extendedspectrum $\beta$-lactamases in the United Kingdom. J Antimicrob Chemother 2008;62:1265-8.

[59] Hansen F, Johansen HK, Ostergaard C, Hansen DS, Littauer P, Homl A, et al. Characterization of carbapenem nonsusceptible Pseudomonas aeruginosa in Denmark: a nationwide prospective study. Microb Drug Resist 2014;20: 22-9.

[60] Jiang X, Zhang Z, Li M, Zhou D, Ruan F, Lu Y. Detection of extended-spectrum $\beta$ lactamases in clinical isolates of Pseudomonas aeruginosa. Antimicrob Agents Chemother 2006;50:2990-5.

[61] Dubois V, Poirel L, Marie C, Arpin C, Nordmann P, Quentin C. Molecular characterization of a novel class 1 integron containing bla $_{\mathrm{GES}-1}$ and a fused product of aac3-Ib/aac6'-Ib' gene cassettes in Pseudomonas aeruginosa. Antimicrob Agents Chemother 2002;46:638-45

[62] Castanheira M, Mendes RE, Walsh TR, Gales AC, Jones RN. Emergence of the extended-spectrum $\beta$-lactamase GES-1 in a Pseudomonas aeruginosa strain from Brazil: report from the SENTRY Antimicrobial Surveillance Program. Antimicrob Agents Chemother 2004;48:2344-5.

[63] Pasteran F, Faccone D, Petroni A, Rapoport M, Galas M, Vazquez M, et al. Novel variant ( bla $_{\mathrm{VIM}-11}$ ) of the metallo- $\beta$-lactamase bla $a_{\mathrm{VIM}}$ family in a GES-1 extended-spectrum- $\beta$-lactamase-producing Pseudomonas aeruginosa clinical isolate in Argentina. Antimicrob Agents Chemother 2005;49:474-5.

[64] Mavroidi A, Tzelepi E, Tsakris A, Miriagou V, Sofianou D, Tzouvelekis LS. An integron-associated $\beta$-lactamase (IBC-2) from Pseudomonas aeruginosa is a variant of the extended-spectrum $\beta$-lactamase IBC-1. J Antimicrob Chemother 2001:48:627-30.

[65] Kotsakis SD, Papagiannitsis CC, Tzelepi E, Legakis NJ, Miriagou V, Tzouvelekis LS. GES-13, a $\beta$-lactamase variant possessing Lys-104 and Asn-170 in Pseudomonas aeruginosa. Antimicrob Agents Chemother 2010;54:1331-3.

[66] Poirel L, Brinas L, Fortineau N, Nordmann P. Integron-encoded GES-type extended-spectrum $\beta$-lactamase with increased activity toward aztreonam in Pseudomonas aeruginosa. Antimicrob Agents Chemother 2005;49:3593-7.

[67] Naas T, Philippon L, Poirel L, Ronco E, Nordmann P. An SHV-derived extended-spectrum $\beta$-lactamase in Pseudomonas aeruginosa. Antimicrob Agents Chemother 1999;43:1281-4.

[68] Mansour W, Dahmen S, Poirel L, Charfi K, Bettaieb D, Boujaafar N, et al. Emergence of SHV-2a extended-spectrum $\beta$-lactamases in clinical isolates of Pseudomonas aeruginosa in a university hospital in Tunisia. Microb Drug Resist 2009;15:295-301.

[69] Chanawong A, M'Zali FH, Heritage J, Lulitanond A, Hawkey PM. SHV-12, SHV5, SHV-2a and VEB-1 extended-spectrum $\beta$-lactamases in Gram-negative bacteria isolated in a university hospital in Thailand. J Antimicrob Chemother 2001;48:839-52

[70] Uemura S, Yokota S, Mizuno H, Sakawaki E, Sawamoto K, Maekawa K, et al. Acquisition of a transposon encoding extended-spectrum $\beta$-lactamase SHV12 by Pseudomonas aeruginosa isolates during the clinical course of a burn patient. Antimicrob Agents Chemother 2010;54:3956-9.

[71] Poirel L, Lebessi E, Castro M, Fèvre C, Foustoujou M, Nordmann P. Nosocomial outbreak of extended-spectrum $\beta$-lactamase SHV-5-producing isolates of Pseudomonas aeruginosa in Athens, Greece. Antimicrob Agents Chemother 2004; $48: 2277-9$

[72] Poirel L, Ronco E, Naas T, Nordmann P. Extended-spectrum $\beta$-lactamase TEM4 in Pseudomonas aeruginosa. Clin Microbiol Infect 1999;5:651-2.

[73] Dubois V, Arpin C, Noury P, Quentin C. Clinical strain of Pseudomonas aeruginosa carrying a bla $\mathrm{TEM}_{\mathrm{TE}} 21$ gene located on a chromosomal interrupted $\mathrm{Tn} A$ type transposon. Antimicrob Agents Chemother 2002;46:3624-6.

[74] Marchandin H, Jean-Pierre H, De Champs C, Sirot D, Darbas H, Perigault PF, et al. Production of a TEM-24 plasmid-mediated extended-spectrum $\beta$ lactamase by a clinical isolate of Pseudomonas aeruginosa. Antimicrob Agents Chemother 2000;44:213-6.

[75] Mugnier P, Dubrous P, Casin I, Arlet G, Collatz E. A TEM-derived extendedspectrum $\beta$-lactamase in Pseudomonas aeruginosa. Antimicrob Agents Chemother 1996:40:2488-93.

[76] Al Naiemi N, Duim B, Bart A. A CTX-M extended-spectrum $\beta$-lactamase in Pseudomonas aeruginosa and Stenotrophomonas maltophilia. J Med Microbiol 2006;55:1607-8.
[77] Picão RC, Poirel L, Gales AC, Nordmann P. Further identification of CTX-M2 extended-spectrum $\beta$-lactamase in Pseudomonas aeruginosa. Antimicrob Agents Chemother 2009;53:2225-6.

[78] Polotto M, Casella T, de Lucca Oliveira MG, Rúbio FG, Nogueira ML, de Almeida $\mathrm{MT}$, et al. Detection of Pseudomonas aeruginosa harboring bla $a_{\mathrm{CXT}-\mathrm{M}-2}, b_{\mathrm{GES}-1}$ and bla $_{\mathrm{GES}-5}$, bla $_{\mathrm{IMP}-1}$ and bla $\mathrm{SPM}-1_{1}$ causing infections in Brazilian tertiary-care hospital. BMC Infect Dis 2012;12:176.

[79] Poirel L, Brinas L, Verlinde A, Ide L, Nordmann P. BEL-1, a novel clavulanic acidinhibited extended-spectrum $\beta$-lactamase, and the class 1 integron In120 in Pseudomonas aeruginosa. Antimicrob Agents Chemother 2005;49:3743-8.

[80] Bogaerts P, Bauraing C, Deplano A, Glupczynski Y. Emergence and dissemination of BEL-1-producing Pseudomonas aeruginosa isolates in Belgium. Antimicrob Agents Chemother 2007:51:1584-5.

[81] Poirel L, Docquier J, De Luca F, Verlinde A, Ide L, Rossolini GM, et al. BEL-2, an extended-spectrum $\beta$-lactamase with increased activity toward expendedspectrum cephalosporins in Pseudomonas aeruginosa. Antimicrob Agents Chemother 2010:54:533-5.

[82] Juan C, Zamorano L, Pérez JL, Ge Y, Oliver A. Activity of a new antipseudomonal cephalosporin, CXA-101 (FR264205), against carbapenem-resistant and multidrug-resistant Pseudomonas aeruginosa clinical strains. Antimicrob Agents Chemother 2010;54:846-51.

[83] Tian GB, Adams-Haduch JM, Bogdanovich T, Wang HN, Doi Y, PME-1, an extended-spectrum $\beta$-lactamase identified in Pseudomonas aeruginosa. Antimicrob Agents Chemother 2011;55:2710-3.

[84] Bae IK, Lee YN, Jeong SH, Hong SG, Lee JH, Lee SH, et al. Genetic and biochemical characterization of GES-5, an extended-spectrum class A $\beta$-lactamase from Klebsiella pneumoniae. Diagn Microbiol Infect Dis 2007;58:465-8.

[85] Bonnin RA, Nordmann P, Potron A, Lécuyer H, Zahar JR, Poirel L. Carbapenemhydrolyzing GES-type extended-spectrum $\beta$-lactamase in Acinetobacter baumannii. Antimicrob Agents Chemother 2011;55:349-54.

[86] Nordmann P, Cuzon G, Naas T. The real threat of Klebsiella pneumoniae carbapenemase-producing bacteria. Lancet Infect Dis 2009;9:228-36.

[87] Naas T, Cuzon G, Villegas MV, Lartigue MF, Quinn JP, Nordmann P. Genetic structures at the origin of acquisition of the $\beta$-lactamase bla $a_{\mathrm{KPC}}$ gene. Antimicrob Agents Chemother 2008;52:1257-63.

[88] Robledo IE, Aquino EE, Santé MI, Santana JL, Otero DM, Leon CF, et al. Detection of KPC in Acinetobacter spp. in Puerto Rico. Antimicrob Agents Chemother 2010;54:1354-7.

[89] Poirel L, Weldhagen GF, Naas T, De Champs C, Dove MG, Nordmann P. GES-2, a class A $\beta$-lactamase from Pseudomonas aeruginosa with increased hydrolysis of imipenem. Antimicrob Agents Chemother 2001:45:2598-603.

[90] Poirel L, Weldhagen GF, De Champs C, Nordmann P. A nosocomial outbreak of Pseudomonas aeruginosa isolates expressing the extended-spectrum $\beta$ lactamase GES-2 in South Africa. J Antimicrob Chemother 2002;49:561-5.

[91] Wang C, Cai P, Chang D, Mi AA. Pseudomonas aeruginosa isolate producing the GES-5 extended-spectrum $\beta$-lactamase. J Antimicrob Chemother 2006;57:1261-2.

[92] Labuschagne J, Weldhagen GF, Ehlers MM, Dove MG. Emergence of class 1 integron-associated GES-5 and GES-5-like extended-spectrum $\beta$-lactamases in clinical isolates of Pseudomonas aeruginosa in South Africa. Int J Antimicrob Agents 2008;31:527-30.

[93] Picão RC, Poirel L, Gales AC, Nordmann P. Diversity of $\beta$-lactamases produced by ceftazidime-resistant Pseudomonas aeruginosa isolates causing bloodstream infections in Brazil. Antimicrob Agents Chemother 2009;53:3908-13.

[94] Iraz M, Duzgun AO, Cicek AC Bonnin RA, Ceylan A, Saral A et al. Characterization of novel VIM carbapenemase, VIM-38, and first detection of GES-5 carbapenem-hydrolyzing $\beta$-lactamases in Pseudomonas aeruginosa in Turkey. Diagn Microbiol Infect Dis 2014;78:292-4.

[95] Bebrone C, Bogaerts P, Delbrück H, Benninck S, Kupper MB, Rezende de Castro $\mathrm{R}$, et al. GES-18, a new carbapenem-hydrolyzing GES-type $\beta$-lactamase from Pseudomonas aeruginosa that contains Ile80 and Ser170 residues. Antimicrob Agents Chemother 2013;57:396-401.

[96] Villegas MV, Lolans K, Correa A, Kattan JN, Lopez JA, Quinn JP. First identification of Pseudomonas aeruginosa isolates producing a KPCtype carbapenem-hydrolyzing $\beta$-lactamase. Antimicrob Agents Chemother 2007;51:1553-5.

[97] Wolter DJ, Khalaf N, Robledo IE, Vazquez GJ, Santé MI, Aquino EE, et al. Surveillance of carbapenem-resistant Pseudomonas aeruginosa isolates from Puerto Rican medical center hospitals: dissemination of KPC and IMP-18 $\beta$ lactamases. Antimicrob Agents Chemother 2009;53:1660-4.

[98] Wolter DJ, Kurpiel PM, Woodford N, Palepou MF, Goering RV, Hanson ND. Phenotypic and enzymatic comparative analysis of the novel KPC variant KPC-5 and its evolutionary variants, KPC-2 and KPC-4. Antimicrob Agents Chemother 2009:53:557-662.

[99] Akpaka PE, Swanston WH, Ihemere HN, Correa A, Torres JA, Tafur JD, et al. Emergence of KPC-producing Pseudomonas aeruginosa in Trinidad and Tobago. J Clin Microbiol 2009;47:2670-1.

[100] Poirel L, Nordmann P, Lagrutta E, Cleary T, Munoz-Price LS. Emergence of KPCproducing Pseudomonas aeruginosa in the United States. Antimicrob Agents Chemother 2010;54:3072.

[101] Ge C, Wei Z, Jiang Y, Shen P, Yu Y, Chen Y. Identification of KPC-2producing Pseudomonas aeruginosa isolates in China. J Antimicrob Chemother 2011;66:1184-6.

[102] Cuzon G, Naas T, Villegas MV, Correa A, Quinn JP, Nordmann P. Wide dissemination of Pseudomonas aeruginosa producing $\beta$-lactamase bla $a_{\mathrm{KPC}-2}$ gene in Colombia. Antimicrob Agents Chemother 2011;55:5350-3. 
[103] Garcia Ramirez D, Nicola F, Zarate S, Relloso S, Smayevsky J, Arduino D. Emergence of Pseudomonas aeruginosa with KPC-type carbapenemase in a teaching hospital: an 8-year study. J Med Microbiol 2013;62:1565-70.

[104] Jacome PR, Alves LR, Cabral AB, Lopes AC, Maciel MA. First report of KPCproducing Pseudomonas aeruginosa in Brazil. Antimicrob Agents Chemother 2012;56:4990.

[105] Nordmann P, Dortet L, Poirel L. Carbapenem resistance in Enterobacteriaceae: here is the storm! Trends Mol Med 2012;18:263-72.

[106] Walsh TR, Toleman MA, Poirel L, Nordmann P. Metallo- $\beta$-lactamases: the quiet before the storm? Clin Microbiol Rev 2005;18:306-25.

[107] Poirel L, Nordmann P. Carbapenem resistance in Acinetobacter baumannii: mechanisms and epidemiology. Clin Microbiol Infect 2006;12:826-36.

[108] Cornaglia G, Riccio ML, Mazzariol A, Lauretti L, Fontana R, Rossolin GM. Appearance of IMP-1 metallo- $\beta$-lactamases in Europe. Lancet 1999;353:899-900.

[109] Shibata N, Doi Y, Yamane K, Yagi T, Kurokawa H, Shibayama K, et al. PCR typing of genetic determinants for metallo- $\beta$-lactamases and integrases carried by Gram-negative bacteria isolated in Japan, with focus on the class 3 integron. J Clin Microbiol 2003;41:5407-13.

[110] Lee K, Lee WG, Uh Y, Ha GY, Cho J, Chong Y. VIM- and IMP-type metallo$\beta$-lactamase-producing Pseudomonas spp. and Acinetobacter spp. in Korean hospitals. Emerg Infect Dis 2003:9:868-71.

[111] Uma Karthika R, Srinivasa Rao R, Sahoo S, Shashikala P, Kanungo R, Jayachandran $S$, et al. Phenotypic and genotypic assays for detecting the prevalence of metallo- $\beta$-lactamases in clinical isolates of Acinetobacter baumannii from a south Indian tertiary care hospital. J Med Microbiol 2009;58:430-5.

[112] Chiu CH, Lee HY, Tseng LY, Chen CL, Chia JH, Su LH, et al. Mechanisms of resistance to ciprofloxacin, ampicillin/sulbactam and imipenem in Acinetobacter baumannii clinical isolates in Taiwan. Int J Antimicrob Agents 2010;35:382-6.

[113] Al-Sweih NA, Al-Hubail M, Rotimi VO. Three distinct clones of carbapenemresistant Acinetobacter baumannii with high diversity of carbapenemases isolated from patients in two hospitals in Kuwait. J Infect Public Health 2012;5:102-8.

[114] Riccio ML, Franceschini N, Boschi L, Caravelli B, Cornaglia G, Fontana R, et al. Characterization of the metallo- $\beta$-lactamase determinant of Acinetobacter baumannii AC-54/97 reveals the existence of bla $a_{\mathrm{IMP}}$ allelic variants carried by gene cassettes of different phylogeny. Antimicrob Agents Chemother 2000;44:1229-35

[115] Chu YW, Afzal-Shah M, Houang ET, Palepou MI, Lyon DJ, Woodford N, et al IMP-4, a novel metallo- $\beta$-lactamase from nosocomial Acinetobacter spp, collected in Hong Kong between 1994 and 1998. Antimicrob Agents Chemother 2001;45:710-4.

[116] Peleg AY, Franklin C, Bell J, Spelman DW. Emergence of IMP-4 metalloB-lactamase in a clinical isolate from Australia. J Antimicrob Chemother 2004;54:699-700.

[117] Koh TH, Sng LH, Wang GC, Hsu LY, Zhao Y. IMP-4 and OXA $\beta$-lactamases in Acinetobacter baumannii from Singapore. J Antimicrob Chemother 2007;59:627-32.

[118] Da Silva GJ, Correia M, Vital C, Ribeiro G, Sousa JC, Leitao R, et al. Molecular characterization of $b a_{\mathrm{IMP}-5}$, a new integron-borne metallo- $\beta$-lactamase gene from an Acinetobacter baumannii nosocomial isolate in Portugal. FEMS Microbiol Lett 2002;215:33-9.

[119] Gales AC, Tognim MC, Reis AO, Jones RN, Sader HS. Emergence of an IMP-like metallo-enzyme in an Acinetobacter baumannii clinical strain from a Brazilian teaching hospital. Diagn Microbiol Infect Dis 2003;45:77-9.

[120] Wang H, Guo P, Sun H, Wang H, Yang Q Chen M, et al. Molecular epidemiology of clinical isolates of carbapenem-resistant Acinetobacter spp. from Chinese hospitals. Antimicrob Agents Chemother 2007;51:4022-8.

[121] Kansakar P, Dorji D, Chongtrakool P, Mingmongkolchai S, Mokmake B, Dubbs P. Local dissemination of multidrug-resistant Acinetobacter baumannii clones in a Thai hospital. Microb Drug Resist 2011;17:109-19.

[122] Yamamoto M, Nagao M, Matsumura Y, Matsushima A, Ito Y, Takakura S, et al. Interspecies dissemination of a novel class 1 integron carrying bla ${ }_{\mathrm{IMP}-19}$ among Acinetobacter species in Japan. J Antimicrob Chemother 2011;66:2480-3.

[123] Tsakris A, Ikonomidis A, Pournaras S, Tzouvelekis LS, Sofianou D, Legakis NJ, et al. VIM-1 metallo- $\beta$-lactamase in Acinetobacter baumannii. Emerg Infect Dis 2006;12:981-3

[124] Mammina C, Bonura C, Aleo A, Calà C, Caputo G, Cataldo MC, et al. Characterization of Acinetobacter baumannii from intensive care units and home care patients in Palermo, Italy. Clin Microbiol Infect 2011:17:E12-5.

[125] Lee K, Yum JH, Yong D, Lee HM, Kim HD, Docquier JD, et al. Novel acquired metallo- $\beta$-lactamase gene, bla SIM-1 $_{1}$, in a class 1 integron from Acinetobac ter baumannii clinical isolates from Korea. Antimicrob Agents Chemothe 2005:49:4485-91

[126] Yong D, Toleman MA, Giske CG, Cho HS, Sundman K, Lee K, et al. Characterization of a new metallo- $\beta$-lactamase gene, bla $a_{\mathrm{NDM}-1}$, and a nove erythromycin esterase gene carried on a unique genetic structure in Klebsiella pneumoniae sequence type 14 from India. Antimicrob Agents Chemother 2009;53:5046-54.

[127] Karthikeyan K, Thirunarayan MA, Krishnan P. Coexistence of bla $a_{\mathrm{OXA}-23}$ with bla $_{\mathrm{NDM}-1}$ and $\operatorname{arm} A$ in clinical isolates of Acinetobacter baumannii from India. J Antimicrob Chemother 2010;65:2253-4.

[128] Krizova L, Bonnin RA, Nordmann P, Nemec A, Poirel L. Characterization of a multidrug-resistant Acinetobacter baumannii strain carrying the bla $a_{\mathrm{NDM}-1}$ and $b_{1} a_{\mathrm{OXA}-23}$ carbapenemase genes from the Czech Republic. J Antimicrob Chemother 2012;67:1550-2.
[129] Pfeifer Y, Wilharm G, Zander E, Wichelhaus TA, Göttig S, Hunfeld KP, et al. Molecular characterization of bla $a_{\mathrm{NDM}-1}$ in an Acinetobacter baumannii strain isolated in Germany in 2007. J Antimicrob Chemother 2011:66:1998-2001.

[130] Bogaerts P, Rezende de Castro R, Roisin S, Deplano A, Huang TD, Hallin M, et al. Emergence of NDM-1-producing Acinetobacter baumannii in Belgium. J Antimicrob Chemother 2012;67:1552-3.

[131] Bonnin RA, Poirel L, Naas T, Pirs M, Seme K, Schrenzel J, et al. Dissemination of New Delhi metallo- $\beta$-lactamase-1-producing Acinetobacter baumannii in Europe. Clin Microbiol Infect 2012;18:E362-5.

[132] Chen Y, Zhou Z, Jiang Y, Yu Y. Emergence of NDM-1-producing Acinetobacter baumannii in China. J Antimicrob Chemother 2011;66:1255-9.

[133] Nakazawa Y, Li R, Tamura T, Hoshina T, Tamura K, Kawano S, et al. A case of NDM-1-producing Acinetobacter baumannii transferred from India to Japan. J Infect Chemother 2013;19:330-2.

[134] Revathi G, Siu LK, Lu PL, Huang LY. First report of NDM-1-producing Acinetobacter baumannii in East Africa. Int J Infect Dis 2013;17:e1255-8.

[135] Pillonetto M, Arend L, Vespero EC, Pelisson M, Chagas TP, Carvalho-Assef AP, et al. First report of NDM-1-producing Acinetobacter baumannii sequence type 25 in Brazil. Antimicrob Agents Chemother 2014;58:7592-4.

[136] Bakour S, Touati A, Bachiri T, Sahli F, Tiouit D, Naim M, et al. First report of 16S rRNA methylase ArmA-producing Acinetobacter baumannii and rapid spread of metallo- $\beta$-lactamase NDM-1 in Algerian hospitals. J Infect Chemother 2014;20:696-701.

[137] Rafei R, Dabboussi F, Hamze M, Eveillard M, Lemarié C, Mallat H, et al. First

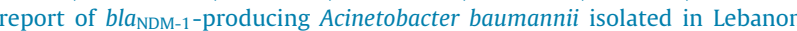
from civilians wounded during the Syrian war. Int J Infect Dis 2014;21:21-3.

[138] Decousser JW, Jansen C, Nordmann P, Emirian A, Bonnin RA, Anais L, et al. Outbreak of NDM-1-producing Acinetobacter baumannii in France, January to May 2013. Euro Surveill 2013;18, pii: 20547

[139] Bonnin RA, Cuzon G, Poirel L, Nordmann P. Multidrug-resistant Acinetobacter baumannii clone, France. Emerg Infect Dis 2013;19:822-3.

[140] Kaase M, Nordmann P, Wichelhaus TA, Gatermann SG, Bonnin RA, Poirel L. NDM-2 carbapenemase in Acinetobacter baumannii from Egypt. J Antimicrob Chemother 2011;66:1260-2.

[141] Espinal P, Fugazza G, Lopez Y, Kasma M, Lerman Y, Malhotra-Kumar S, et al. Dissemination of an NDM-2-producing Acinetobacter baumannii clone in an Israeli rehabilitation center. Antimicrob Agents Chemother 2011;55:5396-8.

[142] Ghazawi A, Sonnevend A, Bonnin RA, Poirel L, Nordmann P, Hashmey R, et al. NDM-2 carbapenemase-producing Acinetobacter baumannii in the United Arab Emirates. Clin Microbiol Infect 2012;18:E34-6.

[143] Espinal P, Poirel L, Carmeli Y, Kaase M, Pal T, Nordmann P, et al. Spread of NDM-2-producing Acinetobacter baumannii in the Middle East. J Antimicrob Chemother 2013;68:1928-30.

[144] Bonnin RA, Poirel L, Nordmann P. New Delhi metallo- $\beta$-lactamase-producing Acinetobacter baumannii: a novel paradigm for spreading antibiotic resistance genes. Future Microbiol 2014:9:33-41.

[145] Queenan AM, Bush K. Carbapenemases: the versatile $\beta$-lactamases. Clin Microbiol Rev 2007;20:440-58

[146] Zhao WH, Chen G, Ito R, Hu ZQ. Relevance of resistance levels to carbapenems and integron-borne bla $a_{\mathrm{IMP}-1}$, bla $_{\mathrm{IMP}-7}$, bla $_{\mathrm{IMP}-10}$ and $b l a_{\mathrm{VIM}-2}$ in clinical isolates of Pseudomonas aeruginosa. J Med Microbiol 2009;58:1080-5.

[147] Martins AF, Zavascki AP, Gaspareto PB, Barth AL. Dissemination of Pseudomonas aeruginosa producing SPM-1-like and IMP-1-like metallo- $\beta$ lactamases in hospitals from southern Brazil. Infection 2007;35:457-60.

[148] Ou TT, Zhang JL, Wang J, Tao J, Yu YS, Chen YG, et al. Evaluation of phenotypic tests for detection of metallo- $\beta$-lactamase-producing Pseudomonas aeruginosa strains in China. J Clin Microbiol 2009;47:1136-42.

[149] Ozgumus OB, Caylan R, Tosun I, Sandalli C, Aydin K, Koksal I. Molecular epidemiology of clinical Pseudomonas aeruginosa isolates carrying IMP-1 metallo- $\beta$-lactamase gene in a university hospital in Turkey. Microb Drug Resist 2007;13:191-8.

[150] Koh TH, Wang GC, Sng LH. Clonal spread of IMP-1-producing Pseudomonas aeruginosa in two hospitals in Singapore. J Clin Microbiol 2004;42:5378-80.

[151] Boonkerd N, Pibalpakdi P. Tiloklurs M, Niumsup PR. Class 1 integron containing metallo $\beta$-lactamase gene bla $a_{\mathrm{IMP}-1}$ in carbapenem-resistant Pseudomonas aeruginosa in Thailand. J Infect Chemother 2009;15:257-61.

[152] Bert F, Vanjak D, Leflon-Guibout V, Mrejen S, Delpierre S, Redondo A, et al IMP-4-producing Pseudomonas aeruginosa in a French patient repatriated from Malaysia: impact of early detection and control measures. Clin Infect Dis 2007;44:764-5.

[153] Peleg AY, Franklin C, Bell JM, Spelman DW. Dissemination of the metallo- $\beta$ lactamase gene bla $a_{\mathrm{IMP}-4}$ among Gram-negative pathogens in a clinical setting in Australia. Clin Infect Dis 2005;41:1549-56.

[154] Brízio A, Conceição T, Pimentel M, Da Silva G, Duarte A. High-level expression of IMP-5 carbapenemase owing to point mutation in the-35 promoter region of class 1 integron among Pseudomonas aeruginosa clinical isolates. Int J Antimicrob Agents 2006;27:27-31.

[155] Ryoo NH, Lee K, Lim JB, Lee YH, Bae IK, Jeong SH. Outbreak by meropenemresistant Pseudomonas aeruginosa producing IMP-6 metallo- $\beta$-lactamase in a Korean hospital. Diagn Microbiol Infect Dis 2009;63:115-7.

[156] Chen Y, Sun M, Wang M, Lu Y, Yan Z. Dissemination of IMP-6-producing Pseudomonas aeruginosa ST244 in multiple cities in China. Eur J Clin Microbiol Infect Dis 2014;33:1181-7.

[157] Gibb AP, Tribuddharat C, Moore RA, Louie TJ, Krulicki W, Livermore DM, et al Nosocomial outbreak of carbapenem-resistant Pseudomonas aeruginosa with a new bla $a_{\mathrm{IMP}}$ allele, bla $a_{\mathrm{IMP}-7}$. Antimicrob Agents Chemother 2002;46:255-8. 
[158] Ho SE, Subramaniam G, Palasubramaniam S, Navaratnam P. Carbapenemresistant Pseudomonas aeruginosa in Malaysia producing IMP-7 $\beta$-lactamase. Antimicrob Agents Chemother 2002;46:3286-7.

[159] Ohlasova D, Kmet V, Niks M. First report of the carbapenem-resistant Pseudomonas aeruginosa producing IMP-7 metallo- $\beta$-lactamase in Slovakia. Int J Antimicrob Agents 2007;30:370-1.

[160] Kouda S, Kuwahara R, Ohara M, Shigeta M, Fujiwara T, Komatsuzawa H, et al. First isolation of bla IMP-7 in a Pseudomonas aeruginosa in Japan. J Infect Chemother 2007;13:276-7.

[161] Hrabák J, Fridrichová M, Stolbová M, Bergerova T, Zemlickova H, Urbaskova P. First identification of metallo- $\beta$-lactamase-producing Pseudomonas aeruginosa in the Czech Republic. Euro Surveill 2009;14:pii:19102.

[162] Hammerum AM, Jakobsen L, Hansen F, Stegger M, Sorensen LA, Andersen PS, et al. Characterisation of an IMP-7-producing ST357 Pseudomonas aeruginosa isolate detected in Denmark using whole genome sequencing. Int J Antimicrob Agents 2015;45:200-1.

[163] Xiong J, Hynes MF, Ye H, Chen H, Yang Y, M'zali F, et al. bla $a_{\text {IMP-9 }}$ and its association with large plasmids carried by Pseudomonas aeruginosa isolates from the People's Republic of China. Antimicrob Agents Chemother 2006;50:355-8.

[164] Iyobe S, Kusadokoro H, Takahashi A, Yomoda S, Okubo T, Nakamura A, et al. Detection of a variant metallo- $\beta$-lactamase, IMP-10, from two unrelated strains of Pseudomonas aeruginosa and an Alcaligenes xylosoxidans strain. Antimicrob Agents Chemother 2002;46:2014-6.

[165] Duljasz W, Gniadkowski M, Sitter S, Wojna A, Jebelean C. First organisms with acquired metallo- $\beta$-lactamases (IMP-13, IMP-22, and VIM-2) reported in Austria. Antimicrob Agents Chemother 2009;53:2221-2.

[166] Pagani L, Colinon C, Migliavacca R, Labonia M, Docquier JD, Nucleo A, et al. Nosocomial outbreak caused by multidrug-resistant Pseudomonas aeruginosa producing IMP-13 metallo- $\beta$-lactamase. J Clin Microbiol 2005;43:3824-8.

[167] Fournier D, Jeannot K, Robert-Nicoud M, Muller E, Cholley P, van der Mee-Marquet N, et al. Spread of the bla $a_{\mathrm{IMP}-13}$ gene in French Pseudomonas aeruginosa through sequence types ST621, ST308 and ST111. Int J Antimicrob Agents 2012;40:571-3.

[168] Naas T, Bogaerts P, Kostyanev T, Cuzon G, Huang TD, Ozsu S, et al. Silent spread of IMP-13-producing Pseudomonas aeruginosa belonging to sequence type 621 in Belgium. J Antimicrob Chemother 2011;66:2178-9.

[169] Piyakul C, Tiyawisutsri R, Boonbumrung K. Emergence of metallo- $\beta$ lactamase IMP-14 and VIM-2 in Pseudomonas aeruginosa clinical isolates from a tertiary-level hospital in Thailand. Epidemiol Infect 2012;140:539-41.

[170] Quinones-Falconi F, Galicia-Velasco M, Marchiaro P, Mussi MA, Ballerini V, Vila AJ. Emergence of Pseudomonas aeruginosa strains producing metallo- $\beta$ lactamases of the IMP-15 and VIM-2 types in Mexico. Clin Microbiol Infect 2010;16:126-31.

[171] Gilarranz R, Juan C, Castillo-Vera J, Chamizo FJ, Artiles F, Alamo I, et al. First detection in Europe of the metallo- $\beta$-lactamase IMP-15 in clinical strains of Pseudomonas putida and Pseudomonas aeruginosa. Clin Microbiol Infect 2013;19:E424-7.

[172] Castanheira M, Deshpande LM, Costello A, Davies TA, Jones TN. Epidemiology and carbapenem resistance mechanisms of carbapenem-non-susceptible Pseudomonas aeruginosa collected during 2009-11 in 14 European and Mediterranean countries. J Antimicrob Chemother 2014;69:1804-14.

[173] Mendes RE, Toleman MA, Ribeiro J, Sader HS, Jones RN, Walsh TR. Integron carrying a novel metallo- $\beta$-lactamase gene, bla IMP-16, and a fused form of aminoglycoside-resistant gene $\operatorname{aac}\left(6^{\prime}\right)-30 / \operatorname{aac}\left(6^{\prime}\right)-I b^{\prime}$ : report from the SENTRY Antimicrobial Surveillance Program. Antimicrob Agents Chemother 2004:48:4693-702.

[174] Hanson ND, Hossain A, Buck L, Moland ES, Thomson KS. First occurrence of a Pseudomonas aeruginosa isolate in the United States producing an IMP metallo- $\beta$-lactamase, IMP-18. Antimicrob Agents Chemother 2006:50:2272-3.

[175] Garza-Ramos U, Tinoco P, Silva-Sanchez J, Morfin-Otero R, Rodriguez-Noriega E, Leon-Garnica G, et al. Metallo- $\beta$-lactamase IMP- 18 is located in a class 1 integron (In96) in a clinical isolate of Pseudomonas aeruginosa from Mexico. Int J Antimicrob Agents 2008;31:78-80.

[176] Pollini S, Antonelli A, Venturelli C, Maradei S, Veggetti A, Bracco S, et al. Acquisition of plasmid-borne bla IMP-19 gene by a VIM-1-positive Pseudomonas aeruginosa of the sequence type 235 epidemic lineage. J Antimicrob Chemother 2013;68:722-4.

[177] Pellegrini C, Mercuri PS, Celenza G, Galleni M, Segatore B, Sacchetti E, et al. Identification of $b a_{\mathrm{IMP}-22}$ in Pseudomonas spp. in urban wastewater and nosocomial environments: biochemical characterization of a new IMP metallo-enzyme variant and its genetic location. J Antimicrob Chemother 2009;63:901-8

[178] Kim MJ, Bae IK, Jeong SH, Kim SH, Song JH, Choi JY. Dissemination of metallo$\beta$-lactamase-producing Pseudomonas aeruginosa of sequence type 235 in Asian countries. J Antimicrob Chemother 2013;68:2820-4.

[179] Koh TH, Khoo CT, Tan TT, Arshad MA, Ang LP, Lau LJ, et al. Multilocus sequence types of carbapenem-resistant Pseudomonas aeruginosa in Singapore carrying metallo- $\beta$-lactamase genes, including the novel bla $_{\mathrm{IMP}-26}$ gene. J Clin Microbiol 2010;48:2563-4.

[180] Jeannot K, Poirel L, Robert-Nicoud M, Cholley P, Nordmann P, Plésiat P. IMP-29, a novel IMP-type metallo- $\beta$-lactamase in Pseudomonas aeruginosa. Antimicrob Agents Chemother 2012;56:2187-90.

[181] Pegg KM, Liu EM, Lacuran AE, Oelschlaeger P. Biochemical characterization of IMP-30, a metallo- $\beta$-lactamase with enhanced activity toward ceftazidime. Antimicrob Agents Chemother 2013;57:5122-6.
[182] Deshpande LM, Davies TA, Blandino G, Nicoletti G, Jones RN, Castanheira M. IMP-33, a new IMP variant detected in Pseudomonas aeruginosa from Sicily. Antimicrob Agents Chemother 2013;57:6401-3.

[183] Pournaras S, Köck R, Mossialos D, Mellmann A, Sakellaris V, Stathopoulos C, et al. Detection of a phylogenetically distinct IMP-type metallo- $\beta$-lactamase, IMP-35, in a CC235 Pseudomonas aeruginosa from the Dutch-German border region (Euregio). J Antimicrob Chemother 2013;68:1271-6.

[184] Tada T, Miyoshi-Akiyama T, Shimada K, Shimojima M, Kirikae T. IMP-43 and IMP-44 metallo- $\beta$-lactamases with increased carbapenemase activities in multidrug-resistant Pseudomonas aeruginosa. Antimicrob Agents Chemother 2013;57:4427-32.

[185] Wang Y, Wang X, Schwarz S, Zhang R, Lei L, Liu X, et al. IMP-45-producing multidrug-resistant Pseudomonas aeruginosa of canine origin. I Antimicrob Chemother 2014;69:2579-81.

[186] Nordmann P, Poirel L. Emerging carbapenemases in Gram-negative aerobes. Clin Microbiol Infect 2002;8:321-31.

[187] Lauretti L, Riccio ML, Mazzariol A, Cornaglia G, Amicosante G, Fontana R, et al. Cloning and characterization of bla $a_{\mathrm{VIM}}$, a new integron-borne metallo$\beta$-lactamase gene from a Pseudomonas aeruginosa clinical isolate. Antimicrob Agents Chemother 1999;43:1584-90.

[188] Corvec S, Poirel L, Decousser JW, Allouch PY, Drugeon H, Nordmann P. Emergence of carbapenem-hydrolysing metallo- $\beta$-lactamase VIM- 1 in Pseudomonas aeruginosa isolates in France. Clin Microbiol Infect 2006;12: $941-2$

[189] Tsakris A, Pournaras S, Woodford N, Palepou MF, Babini GS, Douboyas J, et al. Outbreak of infections caused by Pseudomonas aeruginosa producing VIM-1 carbapenemase in Greece. J Clin Microbiol 2000:38:1290-2.

[190] Mansour W, Poirel L, Bettaieb D, Bouallegue O, Boujaafar N, Nordmann P. Metallo- $\beta$-lactamase-producing Pseudomonas aeruginosa isolates in Tunisia. Diagn Microbiol Infect Dis 2009;64:458-61.

[191] Castanheira M, Bell JM, Turnidge JD, Mathai D, Jones RN. Carbapenem resistance among Pseudomonas aeruginosa strains from India: evidence for nationwide endemicity of multiple metallo- $\beta$-lactamase clones (VIM-2, -5, -6, and -11 and the newly characterized VIM-18). Antimicrob Agents Chemother 2009;53:1225-7.

[192] Pitout JD, Revathi G, Chow BL, Kabera B, Kariuki S, Nordmann P, et al. Metallo$\beta$-lactamase-producing Pseudomonas aeruginosa isolated from a large tertiary centre in Kenya. Clin Microbiol Infect 2008;14:755-9.

[193] Libisch B, Watine J, Balogh B, Gacs M, Muzslay M, Szabo G, et al. Molecular typing indicates an important role for two international clonal complexes in dissemination of VIM-producing Pseudomonas aeruginosa clinical isolates in Hungary. Res Microbiol 2008;159:162-8.

[194] Khosravi Y, Tee Tay S, Vadivelu J. Metallo- $\beta$-lactamase-producing imipenemresistant Pseudomonas aeruginosa clinical isolates in a university teaching hospital in Malaysia: detection of IMP-7 and first identification of IMP-4, VIM-2, and VIM-11. Diagn Microbiol Infect Dis 2010;67:294-6.

[195] Yan JJ, Hsueh PR, Ko WC, Luh KT, Tsai SH, Wu HM, et al. Metallo- $\beta$ lactamases in clinical Pseudomonas isolates in Taiwan and identification of VIM-3, a novel variant of the VIM-2 enzyme. Antimicrob Agents Chemother 2001;45:2224-8.

[196] Libisch B, Gacs M, Csiszár K, Muzslay M, Rókusz L, Füzi M. Isolation of an integron-borne bla ${ }_{\mathrm{VIM}-4}$ type metallo- $\beta$-lactamase gene from a carbapenemresistant Pseudomonas aeruginosa clinical isolate in Hungary. Antimicrob Agents Chemother 2004;48:3576-8.

[197] Castanheira M, Bell JM, Turnidge JD, Mendes RE, Jones RN. Dissemination and genetic context analysis of bla $\mathrm{VIM}_{\mathrm{M}-6}$ among Pseudomonas aeruginosa isolates in Asian-Pacific nations. Clin Microbiol Infect 2009;16:186-9.

[198] Toleman MA, Rolston K, Jones RN, Walsh TR. blaviM-7, an evolutionarily distinct metallo- $\beta$-lactamase gene in a Pseudomonas aeruginosa isolate from the United States. Antimicrob Agents Chemother 2004;48:329-32.

[199] Crespo MP, Woodford N, Sinclair A, Kaufmann ME, Turton J, Glover J, et al. Outbreak of carbapenem-resistant Pseudomonas aeruginosa producing VIM8 , a novel metallo- $\beta$-lactamase, in a tertiary care center in Cali, Colombia. J Clin Microbiol 2004;42:5094-101.

[200] Marchiaro P, Tomatis PE, Mussi MA, Pasteran F, Viale AM, Limansky AS, et al. Biochemical characterization of metallo- $\beta$-lactamase VIM-11 from a Pseudomonas aeruginosa clinical strain. Antimicrob Agents Chemother 2008;52:2250-2

[201] Juan C, Beceiro A, Gutiérrez O, Alberti S, Garau M, Perez JL et al Characterization of the new metallo- $\beta$-lactamase VIM- 13 and its integron-borne gene from a Pseudomonas aeruginosa clinical isolate in Spain. Antimicrob Agents Chemother 2008;52:3589-96.

[202] Mazzariol A, Mammina C, Koncan R, Di Gaetano V, Di Carlo P, Cipolla D, et al. A novel VIM-type metallo- $\beta$-lactamase (VIM-14) in a Pseudomonas aeruginosa clinical isolate from a neonatal intensive care unit. Clin Microbiol Infect 2011;17:722-4.

[203] Schneider I, Keuleyan E, Rasshofer R, Markovska R, Queenan AM, Bauernfeind A. VIM-15 and VIM-16, two new VIM-2-like metallo- $\beta$-lactamases in Pseudomonas aeruginosa isolates from Bulgaria and Germany. Antimicrob Agents Chemother 2008;52:2977-9.

[204] Siarkou VI, Vitti D, Protonotariou E, Ikonomidis A, Sofianou D. Molecular epidemiology of outbreak-related Pseudomonas aeruginosa strains carrying the novel variant bla ${ }_{\mathrm{VIM}-17}$ metallo- $\beta$-lactamase gene. Antimicrob Agents Chemother 2009;53:1325-30.

[205] Riera E, Cabot G, Mulet X, Garcia-Castillo M, del Campo Juan C, et al. Pseudomonas aeruginosa carbapenem resistance mechanisms in Spain: impact 
on the activity of imipenem, meropenem and doripenem. J Antimicrob Chemother 2011;66:2022-7.

[206] El-Mahdy TS. Identification of a novel metallo- $\beta$-lactamase VIM-28 located within unusual arrangement of class 1 integron structure in Pseudomonas aeruginosa from Egypt. Jpn J Infect Dis 2014;67:382-4.

[207] Poirel L, Magalhaes M, Lopes M, Nordmann P. Molecular analysis of metallo- $\beta$-lactamase gene blaspM-1-surrounding sequences from disseminated Pseudomonas aeruginosa isolates in Recife, Brazil. Antimicrob Agents Chemother 2004;48:1406-9.

[208] Gales AC, Menezes LC, Silbert S, Sader HS. Dissemination in distinct Brazilian regions of an epidemic carbapenem-resistant Pseudomonas aeruginosa producing SPM metallo- $\beta$-lactamase. J Antimicrob Chemother 2003:52:699-702

[209] Salabi AE, Toleman MA, Weeks J, Bruderer T, Frei R, Walsh TR. First report of the metallo- $\beta$-lactamase SPM- 1 in Europe. Antimicrob Agents Chemother 2010;54:582.

[210] Toleman MA, Bennett PM, Walsh TR. ISCR elements: novel gene-capturing systems of the 21st century? Microbiol Mol Biol Rev 2006;70:296-316.

[211] Castanheira M, Toleman MA, Jones RN, Schmidt FJ, Walsh TR. Molecular characterization of a $\beta$-lactamase gene, bla $a_{\mathrm{GIM}-1}$, encoding a new subclass of metallo- $\beta$-lactamase. Antimicrob Agents Chemother 2004;48:4654-61.

[212] Wendel AF, Brodner AH, Wydra S, Ressina S, Henrich B, Pfeffer $K$, et al. Genetic characterization and emergence of the metallo- $\beta$-lactamase GIM1 in Pseudomonas spp. and Enterobacteriaceae during a long-term outbreak. Antimicrob Agents Chemother 2013;57:5162-5.

[213] Jovcic B, Lepsanovic Z, Suljagic V, Rackov G, Begovic J, Topisirovic L, et al. Emergence of NDM-1 metallo- - -lactamase in Pseudomonas aeruginosa clinical isolates from Serbia. Antimicrob Agents Chemother 2011;55:3929-31.

[214] Flateau C, Janvier F, Delacour H, Males S, Ficko C, Andriamanantena D, et al. Recurrent pyelonephritis due to NDM- 1 metallo- $\beta$-lactamase producing Pseudomonas aeruginosa in a patient returning from Serbia, France, 2012. Euro Surveill 2012;17:pii:20311.

[215] Janvier F, Jeannot K, Tessé S, Robert-Nicoud M, Delacour H, Rapp C et al. Molecular characterization of $b^{\prime} a_{\mathrm{NDM}-1}$ in a sequence type $235 \mathrm{Pseu}$ domonas aeruginosa isolate from France. Antimicrob Agents Chemothe 2013:57:3408-11.

[216] Khajuria A, Praharaj AK, Kumar M, Grover N. Emergence of NDM-1 in the clinical isolates of Pseudomonas aeruginosa in India. J Clin Diagn Res 2013;7:1328-31.

[217] Carattoli A, Fortini D, Galetti R, Garcia-Fernandez A, Nardi G, Orazi D, et al. Isolation of NDM-1-producing Pseudomonas aeruginosa sequence type ST235 from a stem cell transplant patient in Italy, May 2013. Euro Surveill 2013;18, pii: 20633.

[218] Zafer MM, Amin M, El Mahallawy H, Ashour MS, Al Agamy M. First report of NDM-1-producing Pseudomonas aeruginosa in Egypt. Int J Infect Dis 2014;29:80-1.

[219] Kulkova N, Babalova M, Sokolova J, Krcmery V. First report of New Delhi metallo- $\beta$-lactamase-1-producing strains in Slovakia. Microb Drug Resist 2015;21:117-20.

[220] Edelstein MV Skleenova EN, Shevchenko OV, D'souza JW Tapalski DV Azizov IS, et al. Spread of extensively resistant VIM-2-positive ST235 Pseudomonas aeruginosa in Belarus, Kazakhstan, and Russia: a longitudinal epidemiological and clinical study. Lancet Infect Dis 2013;13:867-76.

[221] Pollini S, Maradei S, Pecile P, Olivo G, Luzzaro F, Docquier JD, et al. FIM-1, a new acquired metallo- $\beta$-lactamase from a Pseudomonas aeruginosa clinica isolate from Italy. Antimicrob Agents Chemother 2013;57:410-6.

[222] Bou G, Martínez-Beltrán J. Cloning, nucleotide sequencing, and analysis of the gene encoding an AmpC $\beta$-lactamase in Acinetobacter baumannii. Antimicrob Agents Chemother 2000;44:428-32.

[223] Héritier C, Poirel L, Nordmann P. Cephalosporinase over-expression resulting from insertion of ISAba1 in Acinetobacter baumannii. Clin Microbiol Infect 2006; $12: 123-30$

[224] Hujer KM, Hamza NS, Hujer AM, Perez F, Helfand MS, Bethel CR, et al Identification of a new allelic variant of the Acinetobacter baumanni cephalosporinase, ADC-7 $\beta$-lactamase: defining a unique family of class $C$ enzymes. Antimicrob Agents Chemother 2005;49:2941-8.

[225] Rodríguez-Martínez JM, Nordmann P, Ronco E, Poirel L. Extended-spectrun cephalosporinase in Acinetobacter baumannii. Antimicrob Agents Chemothe 2010;54:3484-8.

[226] Tian GB, Adams-Haduch JM, Taracila M, Bonomo RA, Wang HN, Doi Y. Extended-spectrum AmpC cephalosporinase in Acinetobacter baumannii: ADC-56 confers resistance to cefepime. Antimicrob Agents Chemother 2011;55:4922-5

[227] Bonomo RA, Szabo D. Mechanisms of multidrug resistance in Acinetobacter species and Pseudomonas aeruginosa. Clin Infect Dis 2006;43:49-56.

[228] Bagge N, Ciofu O, Hentzer M, Campbell JI, Givskov M, Høiby N. Constitutive high expression of chromosomal $\beta$-lactamase in Pseudomonas aeruginosa caused by a new insertion sequence (IS1669) located in $a m p D$. Antimicrob Agents Chemother 2002;46:3406-11.

[229] Rodríguez-Martínez JM, Poirel L, Nordmann P. Extended-spectrum cephalosporinases in Pseudomonas aeruginosa. Antimicrob Agents Chemother 2009;53:1766-71

[230] Rodríguez-Martínez JM, Poirel L, Nordmann P. Molecular epidemiology and mechanisms of carbapenem resistance in Pseudomonas aeruginosa. Antimicrob Agents Chemother 2009;53:4783-8.
[231] Poirel L, Naas T, Nordmann P. Diversity, epidemiology and genetics of class D B-lactamases. Antimicrob Agents Chemother 2010;54:24-38.

[232] Poirel L, Pitout JD, Nordmann P. Carbapenemases: molecular diversity and clinical consequences. Future Microbiol 2007;2:501-12.

[233] Héritier C, Poirel L, Fournier PE, Claverie JM, Raoult D, Nordmann P. Characterization of the naturally occurring oxacillinase of Acinetobacter baumannii. Antimicrob Agents Chemother 2005;49:4174-9.

[234] Turton JF, Ward ME, Woodford N, Kaufmann ME, Pike R, Livermore DM, et al. The role of ISAba1 in expression of OXA carbapenemase genes in Acinetobacter baumannii. FEMS Microbiol Lett 2006;258:72-7.

[235] Corvec S, Poirel L, Naas T, Drugeon H, Nordmann P. Genetics and expression of the carbapenem-hydrolyzing oxacillinase gene $b a_{\mathrm{OXA}-23}$ in Acinetobacter baumannii. Antimicrob Agents Chemother 2007:51:1530-3.

[236] Olaitan AO, Berrazeg M, Fagade OE, Adelowo OO, Alli JA, Rolain JM. Emergence of multidrug-resistant Acinetobacter baumannii producing OXA-23 carbapenemase, Nigeria. Int J Infect Dis 2013;17:e469-70.

237] Merino M, Poza M, Roca I, Barba MJ, Sousa MD, Vila J, et al. Nosocomia outbreak of a multiresistant Acinetobacter baumannii expressing OXA-23 carbapenemase in Spain. Microb Drug Resist 2014;20:259-63.

[238] Afzal-Shah M, Woodford N, Livermore DM. Characterization of OXA-25, OXA26, and OXA-27, molecular class D B-lactamases associated with carbapenem resistance in clinical isolates of Acinetobacter baumannii. Antimicrob Agents Chemother 2001;45:583-8.

[239] Héritier C, Poirel L, Aubert D, Nordmann P. Genetic and functional analysis of the chromosome-encoded carbapenem-hydrolyzing oxacillinase OXA-40 o Acinetobacter baumannii. Antimicrob Agents Chemother 2003;47:268-73.

[240] Lolans K, Rice TW, Munoz-Price LS, Quinn JP. Multicity outbreak of carbapenem-resistant Acinetobacter baumannii isolates producing the carbapenemase OXA-40. Antimicrob Agents Chemother 2006;50:2941-5.

[241] Da Silva GJ, Quinteira S, Bértolo E, Sousa JC, Gallego L, Duarte A, et al Long-term dissemination of an OXA-40 carbapenemase-producing Acinetobacter baumannii clone in the Iberian Peninsula. J Antimicrob Chemother 2004;54:255-8

[242] Tada T, Miyoshi-Akiyama T, Shimada K, Shimojima M, Kirikae T. Dissemination of 16S rRNA methylase ArmA-producing Acinetobacter baumannii and emergence of OXA-72 carbapenemase coproducers in Japan. Antimicrob Agents Chemother 2014;58:2916-20.

[243] de Sá Cavalcanti FL, Almeida AC, Vilela MA, de Morais Junior MA, de Morais MM, Leal-Balbino TC. Emergence of extensively drug-resistant OXA-72-producing Acinetobacter baumannii in Recife, Brazil: risk of clonal dissemination? Diagn Microbiol Infect Dis 2013:77:250-1.

[244] Povilonis J, Seputiene V, Krasauskas R, Juskaite R, Miskinyte M, Suziedelis K et al. Spread of carbapenem-resistant Acinetobacter baumannii carrying a plasmid with two genes encoding OXA-72 carbapenemase in Lithuanian hospitals. I Antimicrob Chemother 2013;68:1000-6.

[245] Goic-Barisic I, Towner KJ, Kovacic A, Sisko-Kraljevic K, Tonkic M, Novak A et al. Outbreak in Croatia caused by a new carbapenem-resistant clone of Acinetobacter baumannii producing OXA-72 carbapenemase. J Hosp Infect 2011;77:368-9.

[246] Poirel L, Marqué S, Héritier C, Segonds C, Chabanon G, Nordmann P. OXA58 , a novel class $D \beta$-lactamase involved in resistance to carbapenems in Acinetobacter baumannii. Antimicrob Agents Chemother 2005;49:202-8.

[247] Héritier C, Dubouix A, Poirel L, Marty N, Nordmann P. A nosocomial outbreak of Acinetobacter baumannii isolates expressing the carbapenem-hydrolysing oxacillinase OXA-58. J Antimicrob Chemother 2005;55:115-8.

[248] Poirel L, Mansour W, Bouallegue O, Nordmann P. Carbapenem-resistant Acinetobacter baumannii isolates from Tunisia producing the OXA-58like carbapenem-hydrolyzing oxacillinase OXA-97. Antimicrob Agents Chemother 2008;52:1613-7.

[249] Higgins PG, Poirel L, Lehmann M, Nordmann P, Seifert H. OXA-143, a novel carbapenem-hydrolyzing class D $\beta$-lactamase in Acinetobacter baumannii. Antimicrob Agents Chemother 2009;53:5035-8

[250] Higgins PG, Pérez-Llarena FJ, Zander E, Fernández A, Bou G, Seifert H. OXA235 , a novel class D $\beta$-lactamase involved in resistance to carbapenems in Acinetobacter baumannii. Antimicrob Agents Chemother 2013;57:2121-6.

[251] Girlich D, Naas T, Nordmann P. Biochemical characterization of the naturally occurring oxacillinase OXA-50 of Pseudomonas aeruginosa. Antimicrob Agents Chemother 2004;48:2043-8

252] Danel F, Hall LM, Gur D, Livermore DM. OXA-15, an extended-spectrum variant of OXA-2 $\beta$-lactamase, isolated from a Pseudomonas aeruginosa strain. Antimicrob Agents Chemother 1997;41:785-90.

[253] Poirel L, Gerome P, De Champs C, Stephanazzi J, Naas T, Nordmann P. Integronlocated oxa-32 gene cassette encoding an extended-spectrum variant of OXA$2 \beta$-lactamase from Pseudomonas aeruginosa. Antimicrob Agents Chemother 2002;46:566-9.

[254] Juan C, Mulet X, Zamorano L, Albertí S, Pérez JL, Oliver A. Detection of the novel extended-spectrum $\beta$-lactamase OXA-161 from a plasmid-located integron in Pseudomonas aeruginosa clinical isolates from Spain. Antimicrob Agents Chemother 2009;53:5288-90.

[255] Hall LM, Livermore DM, Gur D, Akova M, Akalin HE. OXA-11, an extendedspectrum variant of OXA-10 (PSE-2) $\beta$-lactamase from Pseudomonas aeruginosa. Antimicrob Agents Chemother 1993;37:1637-44.

[256] Danel F, Hall LM, Gur D, Livermore DM. OXA-14, another extended-spectrum variant of OXA-10 (PSE-2) B-lactamase from Pseudomonas aeruginosa. Antimicrob Agents Chemother 1995;39:1881-4. 
[257] Danel F, Hall LM, Gur D, Livermore DM. OXA-16, a further extended-spectrum variant of OXA-10 $\beta$-lactamase, from two Pseudomonas aeruginosa isolates. Antimicrob Agents Chemother 1998;42:3117-22.

[258] Danel F, Hall LM, Duke B, Gur D, Livermore DM. OXA-17, a further extended-spectrum variant of OXA-10 $\beta$-lactamase, isolated from Pseudomonas aeruginosa. Antimicrob Agents Chemother 1999;43:1362-6.

[259] Mugnier P, Casin I, Bouthors AT, Collatz E. Novel OXA-10-derived extendedspectrum $\beta$-lactamases selected in vivo or in vitro. Antimicrob Agents Chemother 1998;42:3113-6.

[260] Poirel L, Girlich D, Naas T, Nordmann P. OXA-28, an extended-spectrum variant of OXA-10 $\beta$-lactamase from Pseudomonas aeruginosa and its plasmidand integron-located gene. Antimicrob Agents Chemother 2001;45:447-53.

[261] Liu W, Liu X, Liao J, Zhang Y, Liang X. Identification of bla two novel OXA-type extended-spectrum- $\beta$-lactamases in Pseudomonas aeruginosa, in Hunan Province, China. J Basic Microbiol 2010;50(Suppl. 1):S116-9.

[262] Hocquet $D$, Colomb M, Dehecq $B$, Belmonte $O$, Courvalin $P$, Plésiat $P$, et al. Ceftazidime-hydrolysing $\beta$-lactamase OXA-145 with impaired hydrolysis of penicillins in Pseudomonas aeruginosa. J Antimicrob Chemother 2011;66:1745-50.

[263] Aubert D, Poirel L, Chevalier J, Leotard S, Pages JM, Nordmann P. Oxacillinasemediated resistance to cefepime and susceptibility to ceftazidime in Pseudomonas aeruginosa. Antimicrob Agents Chemother 2001;45:1615-20.

[264] Philippon LN, Naas T, Bouthors AT, Barakett V, Nordmann P. OXA-18, a class D clavulanic acid-inhibited extended-spectrum $\beta$-lactamase from Pseudomonas aeruginosa. Antimicrob Agents Chemother 1997;41:2188-95.

[265] Toleman MA, Rolston K, Jones RN, Walsh TR. Molecular and biochemical characterization of OXA-45, an extended-spectrum class 2 d' $\beta$-lactamase in Pseudomonas aeruginosa. Antimicrob Agents Chemother 2003;47: 2859-63.

[266] Li H, Walsh TR, Toleman MA. Molecular analysis of the sequences surrounding bla $a_{\text {OxA-45 }}$ reveals acquisition of this gene by Pseudomonas aeruginosa via a novel ISCR element, ISCR5. Antimicrob Agents Chemother 2009;53:1248-51.

[267] Sevillano E, Gallego L, Garcia-Lobo JM. First detection of the OXA-40 carbapenemase in Pseudomonas aeruginosa isolates, located on a plasmid also found in Acinetobacter baumannii. Pathol Biol 2009;57:493-5.

[268] El Garch F, Bogaerts P, Bebrone C, Galleni M, Glupczynski Y. OXA-198, an acquired carbapenem-hydrolyzing class D $\beta$-lactamase from Pseudomonas aeruginosa. Antimicrob Agents Chemother 2011;55:4828-33.

[269] Doi Y, Arakawa Y. 16S ribosomal RNA methylation: emerging resistance mechanism against aminoglycosides. Clin Infect Dis 2007:45:88-94.

[270] Doi Y, Wachino J, Arakawa Y. Nomenclature of plasmid-mediated 16S rRNA methylases responsible for panaminoglycoside resistance. Antimicrob Agents Chemother 2008;52:2287-8,

[271] Wachino J, Arakawa Y. Exogenously acquired 16S rRNA methyltransferases found in aminoglycoside-resistant pathogenic Gram-negative bacteria: an update. Drug Resist Updat 2012;15:133-48.

[272] Galimand M, Courvalin P, Lambert T. RmtF, a new member of the aminoglycoside resistance 16S rRNA N7 G1405 methyltransferase family. Antimicrob Agents Chemother 2012;56:3960-2.

[273] Bueno MF, Francisco GR, O'Hara JA, de Oliveira Garcia D, Doi Y. Coproduction of 16S rRNA methyltransferase RmtD or RmtG with KPC-2 and CTX-M group extended-spectrum $\beta$-lactamases in Klebsiella pneumoniae. Antimicrob Agents Chemother 2013;57:2397-400.

[274] Zhou H, Du XX, Yang O, Zhou JY, Yu YS, Li LJ. Study on carbapenemase and 16S rRNA methylase of imipenem-resistant Acinetobacter baumannii. Zhonghua Liu Xing Bing Xue Za Zhi 2009;30:269-72 [in Chinese].

[275] Cho YJ, Moon DC, Jin JS, Choi CH, Lee YC, Lee JC. Genetic basis of resistance to aminoglycosides in Acinetobacter spp. and spread of armA in Acinetobacter baumannii sequence group 1 in Korean hospitals. Diagn Microbiol Infect Dis 2009;64:185-90.

[276] Hong SB, Shin KS, Ha J, Han K. Co-existence of bla OXA-23 $_{\text {and }} \mathrm{arm} A$ in multidrugresistant Acinetobacter baumannii isolated from a hospital in South Korea. J Med Microbiol 2013;62:836-44.

[277] Tada T, Miyoshi-Akiyama T, Kato Y, Ohmagari N, Takeshita N, Hung NV, et al. Emergence of 16S rRNA methylase-producing Acinetobacter baumannii and Pseudomonas aeruginosa isolates in hospitals in Vietnam. BMC Infect Dis 2013;13:251-6.

[278] Yamada Y Suwabe A. Diverse carbapenem-resistance mechanisms in 16S rRNA methylase-producing Acinetobacter baumannii. J Med Microbiol 2013;62:618-22

[279] Doi Y, Adams JM, Yamane K, Paterson DL. Identification of $16 S$ rRNA methylase-producing Acinetobacter baumannii clinical strains in North America. Antimicrob Agents Chemother 2007:51:4209-10.

[280] Karah N, Haldorsen B, Hermansen NO, Tveten Y, Ragnhildstveit E, Skutlaberg DH, et al. Emergence of OXA-carbapenemase- and 16S rRNA methylaseproducing international clones of Acinetobacter baumannii in Norway. J Med Microbiol 2011;60:515-21.

[281] Brigante G, Migliavacca R, Bramati S, Motta E, Nucleo E, Manenti M, et al. Emergence and spread of multidrug-resistant Acinetobacter baumannii clone producing both the carbapenemase OXA-23 and the 16S rRNA methylase ArmA. J Med Microbiol 2012;61:653-61.

[282] Strateva T, Markova B, Marteva-Proevska Y, Ivanova D, Mitov I. Widespread dissemination of multidrug-resistant Acinetobacter baumannii producing OXA-23 carbapenemase and ArmA 16S ribosomal RNA methylase in a Bulgarian university hospital. Braz J Infect Dis 2012;16:307-10.
[283] Aghazadeh M, Rezaee MA, Nahaei MR, Mahdian R, Pajand O, Saffari F, et al. Dissemination of aminoglycoside-modifying enzymes and 16S rRNA methylases among Acinetobacter baumannii and Pseudomonas aeruginosa isolates. Microb Drug Resist 2013;19:282-8.

[284] Yokoyama K, Doi Y, Yamane K, Kurokawa H, Shibata N, Shibayama K, et al. Acquisition of 16S rRNA methylase gene in Pseudomonas aeruginosa. Lancet 2003;362:1888-93.

[285] Yamane K, Doi Y, Yokoyama K, Yagi T, Kurokawa H, Shibata N, et al. Genetic environments of the rmtA gene in Pseudomonas aeruginosa clinical isolates. Antimicrob Agents Chemother 2004;48:2069-74.

[286] Jin JS, Kwon KT, Moon DC, Lee JC. Emergence of 16S rRNA methylase rmtA in colistin-only-sensitive Pseudomonas aeruginosa in South Korea. Int J Antimicrob Agents 2009;33:490-1.

[287] Doi Y, de Oliveira Garcia D, Adams J, Paterson DL. Coproduction of novel 16S rRNA methylase RmtD and metallo- $\beta$-lactamase SPM-1 in a panresistant Pseudomonas aeruginosa isolate from Brazil. Antimicrob Agents Chemother 2007;51:852-6.

[288] Doi Y, Ghilardi AC, Adams J, de Oliveira Garcia D, Paterson DL. High prevalence of metallo- $\beta$-lactamase and 16S rRNA methylase coproduction among imipenem-resistant Pseudomonas aeruginosa isolates in Brazil. Antimicrob Agents Chemother 2007;51:3388-90.

[289] Gurung M, Moon DC, Tamang MD, Kim J, Lee YC, Seol SY, et al. Emergence of $16 \mathrm{~S}$ rRNA methylase gene armA and cocarriage of $b a_{\mathrm{IMP}-1}$ in Pseudomonas aeruginosa isolates from South Korea. Diagn Microbiol Infect Dis 2010;68:468-70.

[290] Magnet S, Courvalin P, Lambert T. Resistance-nodulation-cell divisiontype efflux pump involved in aminoglycoside resistance in Acinetobacter baumannii strain BM4454. Antimicrob Agents Chemother 2001;45: 3375-80.

[291] Damier-Piolle L, Magnet S, Brémont S, Lambert T, Courvalin P. AdeIJK, a resistance-nodulation-cell division pump effluxing multiple antibiotics in Acinetobacter baumannii. Antimicrob Agents Chemother 2008:52: 557-62.

[292] Su XZ, Chen J, Mizushima T, Kuroda T, AbeM. Tsuchiya T. an $\mathrm{H}^{+}$-coupled Acinetobacter baumannii multidrug efflux pump belonging to the MATE family of transporters. Antimicrob Agents Chemother 2005;49:4362-4.

[293] Li XZ, Nikaido H, Poole K. Role of mexA-mexB-oprM in antibiotic efflux in Pseudomonas aeruginosa. Antimicrob Agents Chemother 1995;39: 1948-53.

[294] Poole K, Gotoh N, Tsujimoto H, Zhao Q, Wada A, Yamasaki T, et al. Overexpression of the mexC-mexD-oprJ efflux operon in $n f x B$-type multidrug-resistant strains of Pseudomonas aeruginosa. Mol Microbiol 1996;21:713-24.

[295] Llanes C, Köhler T, Patry I, Dehecq B, van Delden C, Plésiat P. Role of the MexEF-OprN efflux system in low-level resistance of Pseudomonas aeruginosa to ciprofloxacin. Antimicrob Agents Chemother 2011;55:5676-84.

[296] Hawkey P, Finch R. Tigecycline: in-vitro performance as a predictor of clinical efficacy. Clin Microbiol Infect 2007;13:354-62.

[297] Ruzin A, Keeney D, Bradford PA. AdeABC multidrug efflux pump is associated with decreased susceptibility to tigecycline in Acinetobacter calcoaceticus-Acinetobacter baumannii complex. J Antimicrob Chemother 2007:59:1001-4.

[298] Coyne S, Rosenfeld N, Lambert T, Courvalin P, Perichon B. Overexpression of resistance-nodulation-cell division pump AdeFGH confers multidrug resistance in Acinetobacter baumannii. Antimicrob Agents Chemother 2010:54:4389-93.

[299] Coyne S, Courvalin P, Périchon B. Efflux-mediated antibiotic resistance in Acinetobacter spp. Antimicrob Agents Chemother 2011;55:947-53.

[300] Rumbo C, Gato E, López M, Ruiz de Alegría C, Fernández-Cuenca F, MartínezMartínez L, et al. Contribution of efflux pumps, porins, and $\beta$-lactamases to multidrug resistance in clinical isolates of Acinetobacter baumannii. Antimicrob Agents Chemother 2013;57:5247-57.

[301] Reid GE, Grim SA, Aldeza CA, Janda WM, Clark NM. Rapid development of Acinetobacter baumannii resistance to tigecycline. Pharmacotherapy 2007;27:1198-201.

[302] Chen Q, Li X, Zhou H, Jiang Y, Chen Y, Hua X, et al. Decreased susceptibility to tigecycline in Acinetobacter baumannii mediated by a mutation in trm encoding SAM-dependent methyltransferase. J Antimicrob Chemother 2014;69:72-6.

[303] Li J, Rayner CR, Nation RL, Owen RJ, Spelman D, Tan KE, et al. Heteroresistance to colistin in multidrug-resistant Acinetobacter baumannii. Antimicrob Agents Chemother 2006;50:2946-50.

[304] Adams MD, Nickel GC, Bajaksouzian S, Lavender H, Murthy AR, Jacobs MR, et al. Resistance to colistin in Acinetobacter baumannii associated with mutations in the PmrAB two-component system. Antimicrob Agents Chemother 2009;53:3628-34.

[305] Beceiro A, Llobet E, Aranda J, Bengoechea JA, Doumith M, Hornsey M, et al. Phosphoethanolamine modification of lipid A in colistin-resistant variants of Acinetobacter baumannii mediated by the pmrAB two-component regulatory system. Antimicrob Agents Chemother 2011:55:3370-9.

[306] Moffatt JH, Harper M, Harrison P, Hale JD, Vinogradov E, Seemann T, et al. Colistin resistance in Acinetobacter baumannii is mediated by complete loss of lipopolysaccharide production. Antimicrob Agents Chemother 2010;54:4971-7.

[307] Hindler JA, Humphries RM. Colistin MIC variability by method for contemporary clinical isolates of multidrug-resistant Gram-negative bacilli. J Clin Microbiol 2013;51:1678-84. 
[308] Ko KS, Suh JY, Kwon KT, Jung SI, Park KH, Kang CI, et al. High rates of resistance to colistin and polymyxin B in subgroups of Acinetobacter baumannii isolates from Korea. J Antimicrob Chemother 2007;60:1163-7.

[309] Valencia R, Arroyo LA, Conde M, Aldana JM, Torres MJ, Fernandez-Cuenca F et al. Nosocomial outbreak of infection with pan-drug-resistant Acinetobacter baumannii in a tertiary care university hospital. Infect Control Hosp Epidemiol 2009;30:257-63.

[310] Bahador A, Taheri M, Pourakbari B, Hashemizadeh Z, Rostami H, Mansoori N, et al. Emergence of rifampicin, tigecycline, and colistin-resistant Acinetobacter baumannii in Iran; spreading of MDR strains of novel international clone variants. Microb Drug Resist 2013;19:397-406.

[311] Lesho E, Yoon EJ, McGann P, Snesrud E, Kwak Y, Milillo M, et al. Emergence of colistin-resistance in extremely drug-resistant Acinetobacter baumannii containing a novel pmrCAB operon during colistin therapy of wound infections. J Infect Dis 2013;208:1142-51.

[312] Tan CH, Li J, Nation RL. Activity of colistin against heteroresistan Acinetobacter baumannii and emergence of resistance in an in vitro pharmacokinetic/pharmacodynamic model. Antimicrob Agents Chemother 2007;51:3413-5.

[313] Denton M, Kerr K, Mooney L, Keer V, Rajgopal A, Brownlee K, et al. Transmission of colistin-resistant Pseudomonas aeruginosa between patients attending a pediatric cystic fibrosis center. Pediatr Pulmonol 2002;34:257-61.
[314] Landman D, Bratu S, Alam M, Quale J. Citywide emergence of Pseudomonas aeruginosa strains with reduced susceptibility to polymyxin B. J Antimicrob Chemother 2005;55:954-7.

[315] Lee JY, Song JH, Ko KS. Identification of nonclonal Pseudomonas aeruginosa isolates with reduced colistin susceptibility in Korea. Microb Drug Resist 2011;17:299-304

[316] Moskowitz SM, Ernst RK, Miller SI. PmrAB, a two-component regulatory system of Pseudomonas aeruginosa that modulates resistance to cationic antimicrobial peptides and addition of aminoarabinose to lipid A. J Bacteriol 2004;186:575-9.

[317] Barrow K, Kwon DH. Alterations in two-component regulatory systems of phoPQ and pmrAB are associated with polymyxin B resistance in clinical isolates of Pseudomonas aeruginosa. Antimicrob Agents Chemother 2009;53:5150-4

[318] Gutu AD, Sgambati N, Strasbourger P, Brannon MK, Jacobs MA, Haugen E, et al Polymyxin resistance of Pseudomonas aeruginosa phoQ mutants is dependent on additional two-component regulatory systems. Antimicrob Agents Chemother 2013;57:2204-15.

[319] Lee JY, Na IY, Park YK, Ko KS. Genomic variations between colistinsusceptible and -resistant Pseudomonas aeruginosa clinical isolates and their effects on colistin resistance. J Antimicrob Chemother 2014;69: $1248-56$. 